\title{
Simply-supported multi-layered beams for energy harvesting
}

\author{
Rupesh Patel ${ }^{1}$, Yoshikazu Tanaka ${ }^{2}$, Stewart McWilliam ${ }^{1,3}$, Hidemi Mutsuda ${ }^{2}$, Atanas A. \\ Popov $^{1}$
}

\begin{abstract}
This paper develops an analytical model for predicting the performance of simply-supported multi-layered piezoelectric vibrating energy harvesters. The model includes the effects of material and geometric non-linearities, as well as axial pre-tension/compression, and is validated against experimental devices for a large range of base accelerations. Numerical and experimental investigations are performed to understand the benefits of using simplysupported devices compared to cantilevered devices. Comparisons are made in an unbiased manner by tuning the resonant frequency to the same value by modifying the geometry, and the results obtained indicate that simplysupported devices are capable of generating higher voltage levels than cantilever devices. The model is also used to investigate the benefits of using multi-layered devices to improve power density. Depending on harvester composition, power-per-unit-volume of piezoelectric material for a device is increased through the stacking of layers.
\end{abstract}

\section{Keywords}

Energy harvesting materials, Non-linearity, Piezoelectric beam, Multi-layered beam, Axial pre-tension/compression, Performance optimisation

\footnotetext{
${ }^{1}$ Faculty of Engineering, University of Nottingham, Nottingham, NG7 2RD, UK

${ }^{2}$ Division of Energy and Environmental Engineering, Hiroshima University, 1-4-1 Kagamiyama, Higashi-Hiroshima, Japan

${ }^{3}$ Faculty of Engineering, University of Nottingham Malaysia Campus, 43500 Semenyih, Malaysia

Corresponding author:

Rupesh Patel, Room C03 ITRC Building, University Park, Nottingham, NG7 2RD, UK

Email: Rupesh.Patel@nottingham.ac.uk
} 


\section{Introduction}

Vibrational energy harvesting using piezoelectric materials is a well-established research area with work being conducted worldwide. To date, typical devices generate power in the micro- to milli-watt scale and are used as a standalone or supplementary power source for wireless sensor nodes. To be relevant to other applications it is vital that harvesters capable of generating higher levels of energy are developed, and this forms the basic motivation here. The work focuses on designing devices having improved harvesting capabilities without increasing the basic foot-print of the device - this may be necessary in practice due to space limitations. Of particular interest is the choice of boundary condition. Most piezoelectric devices are designed, modelled and manufactured as cantilevers (see, for example, Erturk and Inman (2008); Zhu et al. (2010b); Patel (2012); Jackson et al. (2014); MIDÉ). This form is often justified on the basis that it provides good energy generating potential whilst having relatively low fundamental frequencies. The current work compares the performance of cantilever devices with simply-supported devices.

Previous work on simply-supported beam energy harvesters involved using an axial compressive force as a fundamental frequency tuning mechanism (Leland and Wright (2006)) and for bandwidth improvements (Xu et al. (2013)). Leland and Wright (2006) conducted modelling and experiments on a simply-supported energy harvester with a compressive axial force. Results indicated a $24 \%$ reduction in resonant frequency could be realised compared to the unloaded case whilst maintaining reasonable power levels. Xu et al. (2013) performed a study on simply-supported buckled devices showing that bi-stable phenomena can increase the bandwidth and power by over $300 \%$ through chaotic and periodic snap-through vibration. Although such research has been conducted on simply-supported energy harvesters, to the knowledge of the authors, performance comparisons between devices having different boundary conditions are not available in the open literature. Another point worth noting is that previous modelling work assumes that the device has uniform cross-section and material composition along its length. One feature of the model developed here is that it is capable of analysing beams comprising of several sections, each with differing material composition. This feature allows models to be developed that more closely resemble real-world device manufacture.

The developed model is based on the transfer matrix method and includes axial pre-tension/compression, which can be used to tune the resonant frequency of the device in the presence of geometric and material non-linearities. The model also includes the ability to represent multi-layered devices in which several layers of piezoelectric material are located on either side of the main centre substrate layer. The perceived advantage of multi-layering is to increase the power density of the energy harvester. Zhu et al. (2010a) have investigated a similar concept using a commercial Finite Element (FE) package and experimental testing. A standard cantilever bimorph device was compared to devices with $n$ piezoelectric layers, where the thicknesses of piezoelectric layers were selected to ensure all devices compared had the same overall thickness of piezoelectric material. Results indicated a device with $n=4$ was superior for power generation in comparison to both a device with $n=6$ and a standard bimorph device, $n=2$. The work was furthered in Zhu et al. (2011), where FE predictions showed clear benefits to harvesting power via multi-layered devices, regardless of electrical connection, and particularly if the thickness of the piezoelectric layer remains the same whilst increasing $n$. However, no experimental work was conducted to verify their findings. Zhang et al. (2013) more recently undertook a similar study on a unimorph energy harvester with multiple layers of piezoelectric material. Reported findings indicated that a device with 22 layers outperforms a device with a single layer by a factor of 5 to 400 depending on the load resistance. However, this is a weak conclusion; the comparison seemed to be unfair as a single layered device naturally has a larger optimum load resistance - higher than the maximum $100 \mathrm{k} \Omega$ load resistance used. Additionally, the developed model was unable to correctly predict experimental results. In the current work, the developed analytical model for multi-layers includes the influence of geometric and materials non-linearities, which are known to be important when predicting the performance of piezoelectric devices under high levels of base acceleration (see, for example, Stanton et al. (2012); Patel et al. (2014)). 


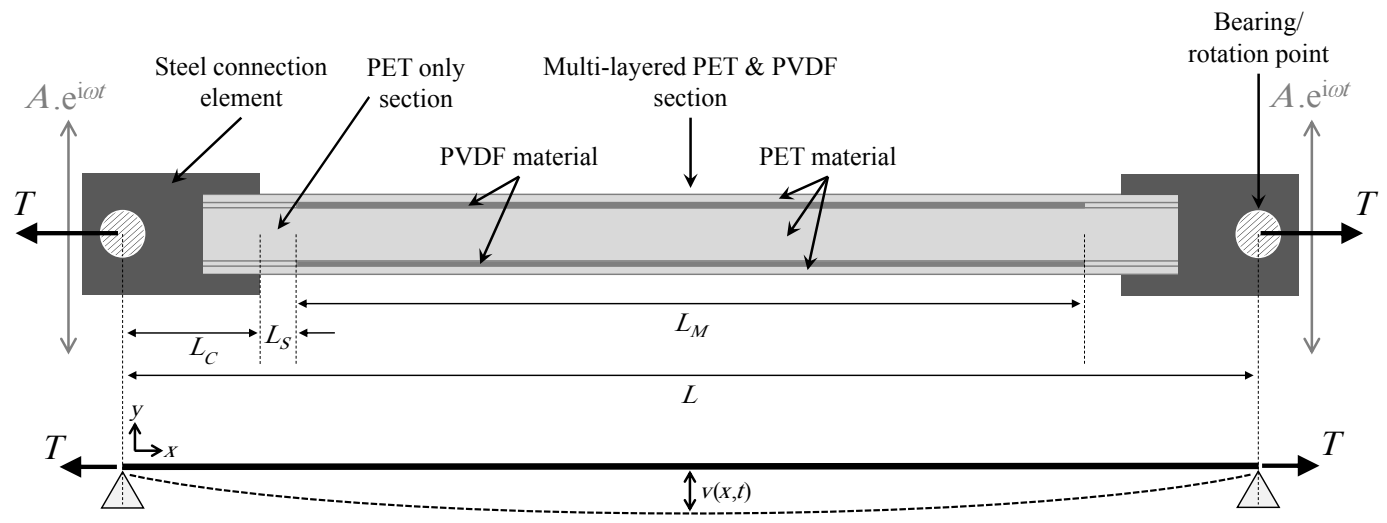

Figure 1. Representation of the simply-supported structure where the energy harvester is a standard bimorph (side view of the device).

The layout and structure of the paper is as follows. The "System Modelling" section develops a model for a simplysupported beam energy harvester, including the presence of axial pre-tension/compression. The transfer matrices are derived, followed by the governing mechanical and electrical equations for multi-layered harvesters, including the effects of material and geometric non-linearity. The "Model Validation" section outlines the experimental procedure used to validate the theoretical model. A range of multi-layered harvesters are considered and excited over a large range of acceleration levels. Testing is also undertaken with device layers in series and parallel connections. The "Model Utilisation" section presents the application of the model to: (i) compare devices with differing boundary conditions; and, (ii) compare devices with various numbers of piezoelectric layers. The key during comparisons will be to ensure devices possess identical natural frequencies thereby allowing unbiased comparisons. Drawing from findings, recommendations are made regarding energy harvester support conditions and design.

\section{System modelling}

Figure 1 shows a schematic representation of the simply-supported beam considered in this study. It is made up of three materials, steel for the connecting elements, polyethylene terephthalate (PET) for the substrate layers and polyvinylidene fluoride (PVDF) for the piezoelectric layers. Subscript ' $C$ ' refers to connecting sections made from steel, subscript ' $S$ ' refers to sections comprising of only PET and subscript ' $M$ ' refers to multi-layered sections made from both PET and PVDF materials.

The device is simply-supported and free to rotate at the bearing/rotation points indicated in Figure 1 and is assumed to be excited through harmonic base motion, $A \mathrm{e}^{\mathrm{i} \omega t}$, where $A$ and $\omega$ are the base acceleration amplitude and excitation frequency, respectively.

\section{Transfer matrices with axial forcing}

The transfer matrix (TM) method can be used to determine the natural frequencies and mode shapes of structures comprising of several sections with differing composition, such as that shown in Figure 1. In essence, TMs relate displacements and forces on the right side of an element to those on the left side of the element, see Pestel and Leckie (1963) for further general details. In this section the derivation of such a TM which includes axial forcing will be presented. 
The governing modal equation for a beam under tension/compression is (Bokaian $(1988,1990)$ ):

$$
E I \frac{\mathrm{d}^{4} v(x)}{\mathrm{d} x^{4}} \pm T \frac{\mathrm{d}^{2} v(x)}{\mathrm{d} x^{2}}-\rho A \omega_{r}^{2} v(x)=0,
$$

where $v(x)$ is the transverse deflection at position $x$ from the left end, and $\omega_{r}$ is the natural frequency. $E I$ and $\rho A$ are the flexural rigidity and mass-per-unit-length of the beam, respectively, which are section dependant and their definitions for multi-layered composite sections can be found in the "Multi-layered device models" section. $T$ is the applied axial force where a +ve sign refers to compression and a -ve sign to tension. The solution to Equation (1) for a mode is expressed as:

$$
v(x)=c_{1} \sinh (M(x / l))+c_{2} \cosh (M(x / l))+c_{3} \sin (N(x / l))+c_{4} \cos (N(x / l))
$$

where $c_{1}$ to $c_{4}$ are constants to be determined and $l$ is the beam length. $M$ and $N$ are defined as (Bokaian (1988, 1990)):

$$
\begin{aligned}
& M=\left( \pm F+\sqrt{F^{2}+\Omega^{2}}\right)^{1 / 2} \\
& N=\left(\mp F+\sqrt{F^{2}+\Omega^{2}}\right)^{1 / 2}
\end{aligned}
$$

where $F=T l^{2} / 2 E I$ is the dimensionless forcing parameter and $\Omega=\omega_{r} l^{2} \sqrt{(\rho A) /(E I)}$ is the dimensionless wave number.

As mentioned, the TM relates displacements and forces on the left end of a beam to the right end, i.e. $[v(l) \theta(l) M(l) F(l)]^{\prime}=\mathbf{U}(l) \cdot[v(0) \theta(0) M(0) F(0)]^{\prime}$, where $\theta$ is the slope, $M$ is the moment, $F$ is the shear force, and $\mathbf{U}(l)$ is the TM. In-order to calculate $\mathbf{U}(l)$, the initial step is to obtain expressions for the first, second and third derivatives of Equation (2). Upon substitution of $x=0$ and $x=l$ into Equation (2) and its three derivatives, one has eight equations. These equations can be used to eliminated constants $c_{1}$ to $c_{4}$ and express $v(l), \theta(l), M(l)$ and $F(l)$ in terms of combinations of $v(0), \theta(0), M(0)$ and $F(0)$ - in essence this is the TM and it is provided below:

$$
\mathbf{U}(l)=\left[\begin{array}{cccc}
H_{1} & l H_{2}+l^{3} \frac{H_{4} T}{E I} & l^{2} \frac{H_{3}}{E I} & l^{3} \frac{H_{4}}{E I} \\
\frac{H_{4} N^{2} M^{2}}{l} & H_{1}+l^{2} \frac{H_{3} T}{E I} & l \frac{H_{5}}{E I} & l^{2} \frac{H_{3}}{E I} \\
\frac{H_{3} E I N^{2} M^{2}}{l^{2}} & \frac{H_{4} E I N^{2} M^{2}}{l}+l H_{5} T & H_{6} & l H_{5} \\
U_{41} & \frac{H_{8}}{l}-T l \frac{H_{5}}{E I} & H_{6}-T l^{2} \frac{H_{3}}{E I}
\end{array}\right],
$$

where, for compactness, $U_{41}$ and $U_{42}$ are given by:

$$
\begin{aligned}
U_{41} & =\frac{H_{7} E I}{l^{3}}-T \frac{H_{4} N^{2} M^{2}}{l}, \\
U_{42} & =\frac{H_{3} E I N^{2} M^{2}}{l^{2}}+T\left(H_{6}-H_{1}-l^{2} \frac{H_{3} T}{E I}\right),
\end{aligned}
$$

and the definitions of constants $H_{1}$ to $H_{8}$ are provided in the "Constants in the transfer matrix" Appendix. 


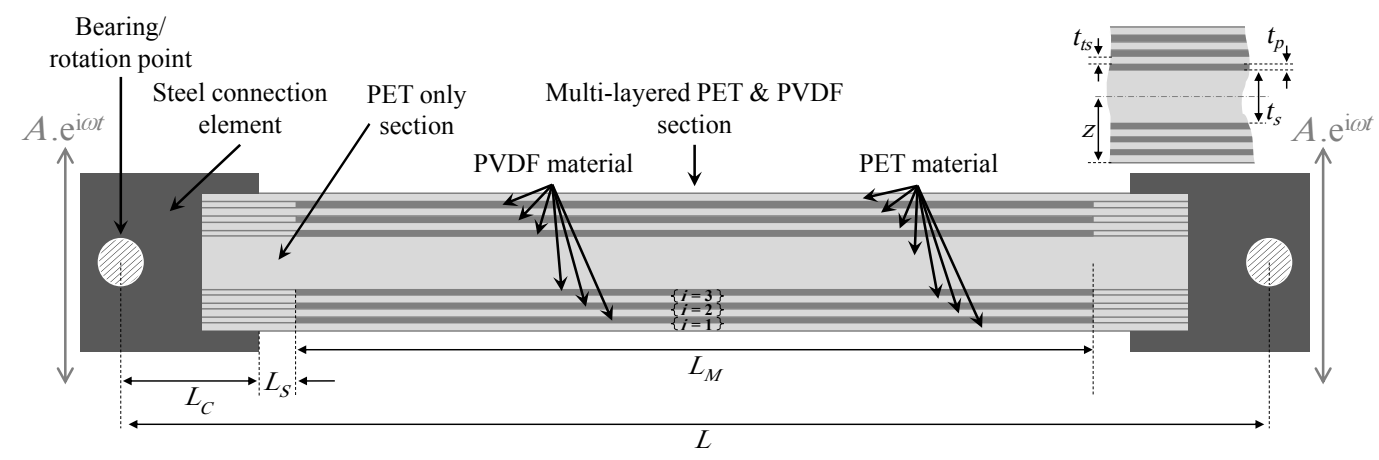

Figure 2. Representation of a multi-layered simply-supported structure. In this example $n$, the number of thin PET/PVDF layers, is 6 .

Equation (5) provides the transfer matrix for a section of beam where $E I$ and $\rho A$ remain constant. Suppose our beam comprises of two sections each with differing lengths, $E I$ and $\rho A$ :

$$
\begin{aligned}
& {[v(l) \theta(l) M(l) F(l)]_{1}^{\prime}=\mathbf{U}_{\mathbf{1}}\left(l_{l}\right) \cdot[v(0) \theta(0) M(0) F(0)]_{1}^{\prime}} \\
& {[v(l) \theta(l) M(l) F(l)]_{2}^{\prime}=\mathbf{U}_{\mathbf{2}}\left(l_{2}\right) \cdot[v(0) \theta(0) M(0) F(0)]_{2}^{\prime}}
\end{aligned}
$$

As a result of continuity, the displacements and forces where the two sections meet are related thus:

$$
[v(l) \theta(l) M(l) F(l)]_{1}^{\prime}=[v(0) \theta(0) M(0) F(0)]_{2}^{\prime},
$$

Equations (8) and (9) can be used to obtain an overall TM for the complete system, which relates displacements and force at the two extremities of the beam, and in this case is given by $\mathbf{U}_{\text {overall }}=\mathbf{U}_{\mathbf{2}}\left(l_{2}\right) \mathbf{U}_{\mathbf{1}}\left(l_{1}\right)$. Extending this logic to the five-section beam shown in Figure 1, the following multiplication is used to obtain the overall TM:

$$
\mathbf{U}_{\text {overall }}=\mathbf{U}_{\mathbf{C}}\left(L_{C}\right) \mathbf{U}_{\mathbf{S}}\left(L_{S}\right) \mathbf{U}_{\mathbf{M}}\left(L_{M}\right) \mathbf{U}_{\mathbf{S}}\left(L_{S}\right) \mathbf{U}_{\mathbf{C}}\left(L_{C}\right)
$$

The four boundary conditions are now applied to extract the relevant $2 \times 2$ matrix for determinant calculation and hence natural frequency extraction. For a simply-supported beam where the deflection and moment are zero at the boundaries, $2 \times 2$ matrix $\left[\mathbf{U}_{\text {overall }}(1,2) \mathbf{U}_{\text {overall }}(1,4) ; \mathbf{U}_{\text {overall }}(3,2) \mathbf{U}_{\text {overall }}(3,4)\right]$ is used. Once the natural frequency has been calculated the mode shape, $W_{r}(x)$, of the entire system can be estimated and mass normalised for use in the equations of motion in the "Overall energy harvester equations of motion" section.

\section{Multi-layered device models}

The multi-layered devices considered are comprised of a core substrate layer with one or more active piezoelectric material and thin substrate layer stacks on either side of this core substrate layer, see Figure 2.

The number of layers of PVDF and thin PET is defined by $n$ which must be an even number for a symmetric arrangement, e.g. $n=6$ in Figure 2. For the composite multi-layered section of such devices the formulation of flexural rigidity, mass and electrical equations from circuit representation is required. The mass-per-unit-length for a multi-layered section can be expressed as:

$$
(\rho A)_{M}=\rho_{s} A_{s}+n \rho_{p} A_{p}+n \rho_{s} A_{t s}
$$




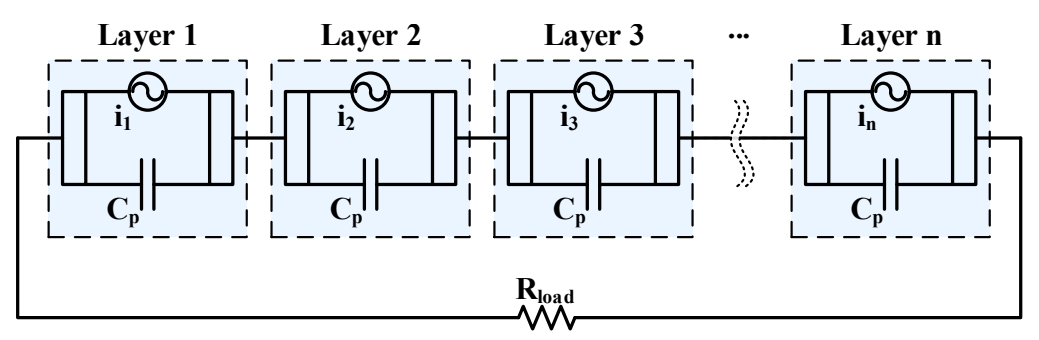

Figure 3. Layers in series connection

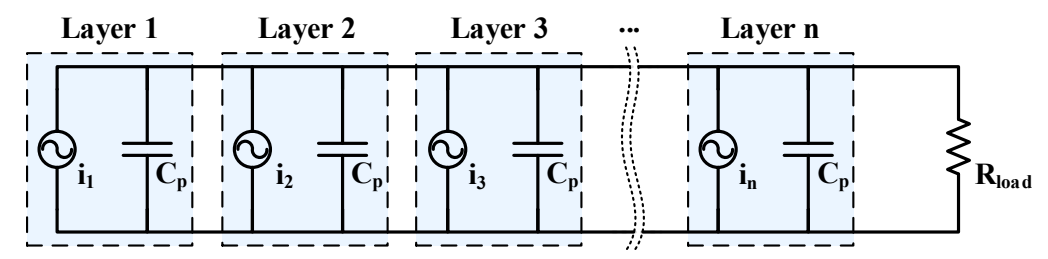

Figure 4. Layers in parallel connection

where subscripts ' $s$ ', ' $p$ ' and ' $t s$ ' refer to the core substrate layer, piezoelectric layer and thinner substrate layer, respectively. $A$ is the cross-sectional area and $\rho$ the material density.

The flexural rigidity of a multi-layered section can be expressed as:

$$
(E I)_{M}=E_{s} I_{z s}+2 E_{p} I_{z p}+2 E_{s} I_{z t s}
$$

where $E$ is the Young's modulus and $I_{z s}, I_{z p}$ and $I_{z t s}$ are second moments of area. The second moments of area are calculated using the parallel axis theorem:

$$
\begin{aligned}
I_{z s} & =\left(b_{s} t_{s}^{3}\right) / 12+A_{s}\left(n / 2\left(t_{t s}+t_{p}\right)+t_{s} / 2-z\right)^{2} \\
I_{z p} & =\sum_{i=1}^{n / 2}\left(b_{p} t_{p}^{3}\right) / 12+A_{p}\left(t_{t s}+(i-1)\left(t_{t s}+t_{p}\right)+t_{p} / 2-z\right)^{2}, \\
I_{z t s} & =\sum_{i=1}^{n / 2}\left(b_{t s} t_{t s}^{3}\right) / 12+A_{t s}\left((i-1)\left(t_{t s}+t_{p}\right)+t_{t s} / 2-z\right)^{2}
\end{aligned}
$$

where layering notation and numbering, $i$, is provided in Figures 2 and 19, $t$ and $b$ represent layer thickness and width, respectively, and $z$ is the distance from the base to the neutral axis, see Figure 2.

It is also important to consider electrical wiring for such multi-layered devices. Figures 3-5 show electrical circuit representations for layers in either a series, parallel or combined series-parallel connection, respectively. For the combined series-parallel connection, all the upper layers in a device are connected in series and separately, all lower layers are connected in series, and then a parallel connection is made between upper and lower layers.

For a series connection between all layers, Figure 3, the electrical equation can be expressed as:

$$
C_{p} \frac{\mathrm{d} V(t)}{\mathrm{d} t}+\frac{n V(t)}{R_{\mathrm{load}}}=2 \sum_{i=1}^{n / 2} i(x, t)
$$




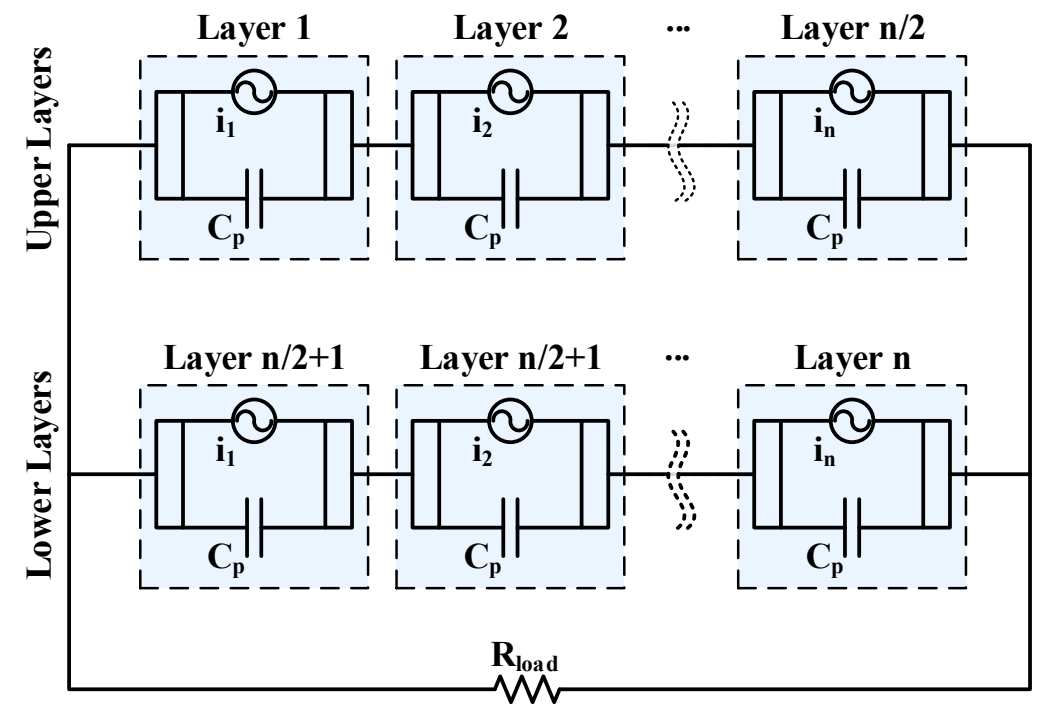

Figure 5. Layers in combined series-parallel connection

where $C_{p}$ is the capacitance of an individual layer, $i(x, t)$ is the current generated by an individual layer and $V(t)$ is the total voltage across resistance $R_{\text {load }}$. The assumption here is that all layers have the same capacitance which is valid provided all piezoelectric layers are manufactured from the same material and with the same dimensions. Similarly for a parallel connection between layers, Figure 4, one has:

$$
C_{p} \frac{\mathrm{d} V(t)}{\mathrm{d} t}+\frac{V(t)}{n R_{\text {load }}}=\frac{2 \sum_{i=1}^{n / 2} i(x, t)}{n}
$$

and for a combined series-parallel connection, Figure 5, one has:

$$
C_{p} \frac{\mathrm{d} V(t)}{\mathrm{d} t}+\frac{n V(t)}{2 R_{\mathrm{load}}}=\sum_{i=1}^{n / 2} i(x, t) .
$$

\section{Overall energy harvester equations of motion}

The non-linear model previously developed by Patel et al. (2016) is seen as a robust and transferable model and is therefore used here to obtain the governing equations of motion. The inclusion of material non-linearity for both piezoelectric and substrate layers, in addition to geometric non-linearity, is important as in some applications, for example engine vibration, the device can be subjected to high levels of acceleration in excess of $20 \mathrm{~ms}^{-2}$. It is assumed that the steel connection elements possess no material non-linearity; this is sensible when considering the material composition of the whole device. Material non-linearity is realised by including odd-power higher order (3rd and 5th) terms in substrate and piezoelectric material constitutive equations (Patel et al. (2016)):

$$
\begin{aligned}
& \sigma_{11}^{s}=E_{s} \varepsilon_{11}^{s}+\mu_{s 1}\left(\varepsilon_{11}^{s}\right)^{3}+\mu_{s 2}\left(\varepsilon_{11}^{s}\right)^{5} \\
& \sigma_{11}^{p}=E_{p} \varepsilon_{11}^{p}+\mu_{p 1}\left(\varepsilon_{11}^{p}\right)^{3}+\mu_{p 2}\left(\varepsilon_{11}^{p}\right)^{5}-E_{p} d_{31} E_{\text {field }}-\mu_{p 3} \varepsilon_{11}^{p} E_{\text {field }}
\end{aligned}
$$


and

$$
D_{3}=E_{p} d_{31} \varepsilon_{11}^{p}+\mu_{p 3}\left(\varepsilon_{11}^{p}\right)^{2}+\varepsilon_{33} E_{\text {field }},
$$

where $\sigma_{11}$ is the axial stress, $\varepsilon_{11}$ is the axial strain, $D_{3}$ is the electric displacement and $E_{\text {field }}$ the electric field strength. $d_{31}$ is a piezoelectric material constant, and $\varepsilon_{33}$ is the material permittivity. $\mu_{s 1}, \mu_{s 2}, \mu_{p 1}, \mu_{p 2}$ and $\mu_{p 3}$ represent coefficients of material non-linearity, and are found through experimental testing.

The non-linear equations of motion, governing the mechanical and electrical response of the harvester, will be obtained by applying the extended Hamilton's principle to a combination of the system Lagrangian and the external work acting on the system:

$$
\int_{t 1}^{t 2} \delta(\mathcal{L}+\mathcal{W}) \mathrm{d} t=0
$$

where the Lagrangian is defined as the difference between kinetic energy, $T$, and potential energy, $U$ :

$$
\mathcal{L}=T-U,
$$

and the external work results from base excitation, $\ddot{w}_{b}(t)$, and the electric potential energy:

$$
\mathcal{W}=\left(\iiint_{V_{s}} \rho_{s} v \mathrm{~d} V_{\text {total }}+\iiint_{V_{p}} \rho_{p} v \mathrm{~d} V_{\text {total }}\right) \ddot{w}_{b}(t)-\mathrm{V}(\mathrm{t}) \mathrm{q}(\mathrm{t}) .
$$

$q(t)$ is the electric charge generated by the energy harvester.

The kinetic energy, $T$, of the system can be expressed as:

$$
T=\frac{1}{2} \int_{0}^{L} m(s)\left(\dot{u}^{2}+\dot{v}^{2}\right) \mathrm{d} s,
$$

where the $m(s)$ is the mass-per-unit-length, and $u$ and $v$ are the longitudinal and transverse deflections, respectively. The inextensibility condition, Equation (27), is then used to relate the longitudinal slope to the transverse slope, thus eliminating $u$ and providing the following final expression for kinetic energy:

$$
\begin{gathered}
T=\frac{1}{2} \int_{0}^{L} m(s)\left[\left(-\int_{0}^{s} v^{\prime} \dot{v}^{\prime}\right)^{2}+\dot{v}^{2}\right] \mathrm{d} s, \\
u^{\prime} \approx-\frac{1}{2}\left(v^{\prime}\right)^{2} .
\end{gathered}
$$

Considering inextensibility, the potential energy, $U$, of the system can be expressed as:

$$
U=\frac{1}{2} \int_{0}^{L} \iint_{A}\left(\sigma_{11} \varepsilon_{11}\right) \mathrm{d} A \mathrm{~d} s-\frac{1}{2} \int_{L_{C}+L_{S}}^{L_{C}+L_{S}+L_{M}} \iint_{A_{p}} E_{\text {field }}(t) D_{3} \mathrm{~d} A \mathrm{~d} s,
$$


where contributions to the first term are calculated for all segments along the beam, and contributions to the second term only arise from the multi-layer section in Figure 1. Using Equations (19)-(21), the potential energy is found to be:

$$
\begin{aligned}
U=\frac{1}{2} \int_{0}^{L} & \left\{\left(K_{1}(s)-K_{5}(s) 2 \sum_{i=1}^{n / 2} V_{i}(t)\right)\left(\left(v^{\prime \prime}\right)^{2}+\left(v^{\prime \prime} v^{\prime}\right)^{2}\right)+K_{2}(s)\left(v^{\prime \prime}\right)^{4}\right. \\
& \left.+K_{3}(s)\left(v^{\prime \prime}\right)^{6}-K_{4}(s)\left(v^{\prime \prime}+\frac{1}{2} v^{\prime \prime}\left(v^{\prime}\right)^{2}\right) 2 \sum_{i=1}^{n / 2} V_{i}(t)-K_{6}(s) 2 \sum_{i=1}^{n / 2} V_{i}^{2}(t)\right\} \mathrm{d} s,
\end{aligned}
$$

where constants $K_{1}$ to $K_{6}$ are defined in the "Potential energy expression constants" Appendix. For a similar calculation procedure for piezoelectric energy harvesters, provided in a step-by-step manner, albeit for a unimorph device, the reader is directed to Patel (2012).

Now that expressions for external work, kinetic energy and potential energy have been obtained, Equation (22) is applied. To avoid derivational repetition, only the final governing equations of motion are provided in this paper and the reader is referred to Patel (2012) and Patel et al. (2014) for procedural details:

$$
\begin{aligned}
\ddot{\eta}_{r}(t) & +2 \gamma_{r} \omega_{r} \dot{\eta}_{r}(t)+\omega_{r}^{2} \eta_{r}(t)+\left(C_{r}^{n 1}+C_{r}^{n 2}\right) \eta_{r}^{3}(t)+C_{r}^{n 3} \eta_{r}^{5}(t)+C_{r}^{n 4} \ddot{\eta}_{r}(t) \eta_{r}^{2}(t)+C_{r}^{n 5} \dot{\eta}_{r}^{2}(t) \eta_{r}(t) \\
& -2 \sum_{i=1}^{n / 2}\left(C_{r}^{n 6}\right)_{i} V_{i}(t)-2 \sum_{i=1}^{n / 2} C_{r}^{n 7} \eta_{r}(t) V_{i}(t)+2 \sum_{i=1}^{n / 2}\left(C_{r}^{n 8}\right)_{i} \eta_{r}^{2}(t) V_{i}(t)-2 \sum_{i=1}^{n / 2} C_{r}^{n 9} \eta_{r}^{3}(t) V_{i}(t)=C_{r}^{n 10} \ddot{w}_{b}(t)
\end{aligned}
$$

and

$$
C_{r}^{n 11} \eta_{r}(t) \dot{\eta}_{r}(t)+C_{r}^{n 12} \eta_{r}^{3}(t) \dot{\eta}_{r}(t)+2 \sum_{i=1}^{n / 2}\left(C_{r}^{n 13}\right)_{i} \dot{\eta}_{r}(t)+2 \sum_{i=1}^{n / 2}\left(C_{r}^{n 14}\right)_{i} \eta_{r}^{2}(t) \dot{\eta}_{r}(t)+C_{r}^{n 15} \dot{V}(t)+\frac{V(t)}{R_{\mathrm{eq}}}=0
$$

$C_{r}^{n 1}-C_{r}^{n 15}$ are time-independent constants defined in the "Constants in the governing equations of motion" Appendix, and $\eta_{r}(t)$ is the time-dependant generalised co-ordinate for the $r^{\text {th }}$ mode. $\gamma_{r}$ and $\omega_{r}$ represent mechanical damping ratio and natural frequency respectively and $\ddot{w}_{b}(t)$ is the base acceleration. Note several differences between Equations (30) and (31) and those presented in Patel et al. (2016):

1. The load resistance, $R_{\text {load }}$, is replaced with $R_{\text {eq }}$. For a series connection between layers $R_{\text {eq }}=R_{\text {load }} / n$, for a parallel connection $R_{\text {eq }}=n R_{\text {load }}$, and for the series-parallel combination $R_{\text {eq }}=2 R_{\text {load }} / n$.

2. Summations are included to account for the contribution of all piezoelectric layers in multi-layered designs.

3. The term $V_{i}(t)$ is present in Equation (30) and represents the voltage across the $i^{\text {th }}$ piezoelectric layer from the base of the device and depends on the electrical connection between layers. If in parallel arrangement, this is simply equal to $V(t)$. However, if in series arrangement, the solution to $C_{p} \dot{V}_{i}(t)=\dot{\eta}_{r}(t)\left(C_{r}^{n 13}\right)_{i}-V(t) / R$ provides approximations for $V_{i}(t)$. Lastly, if in a series-parallel arrangement, approximations for $V_{i}(t)$ are obtained from the solution to $C_{p} \dot{V}_{i}(t)=\dot{\eta}_{r}(t)\left(C_{r}^{n 13}\right)_{i}-V(t) / 2 R$. This calculation of voltages for individual layers is required in order to accurately predict the coupling effects between the system's electrical and mechanical aspects.

The modal information extracted from the transfer matrix procedure can be used in Equations (30) and (31) to solve for $\eta_{r}$ and $V$. The traverse deflection as a function of distance along the beam is then obtained through:

$$
v_{\text {rel }}(x, t)=\sum_{r=1}^{\infty} W_{r}(x) \eta_{r}(t)
$$




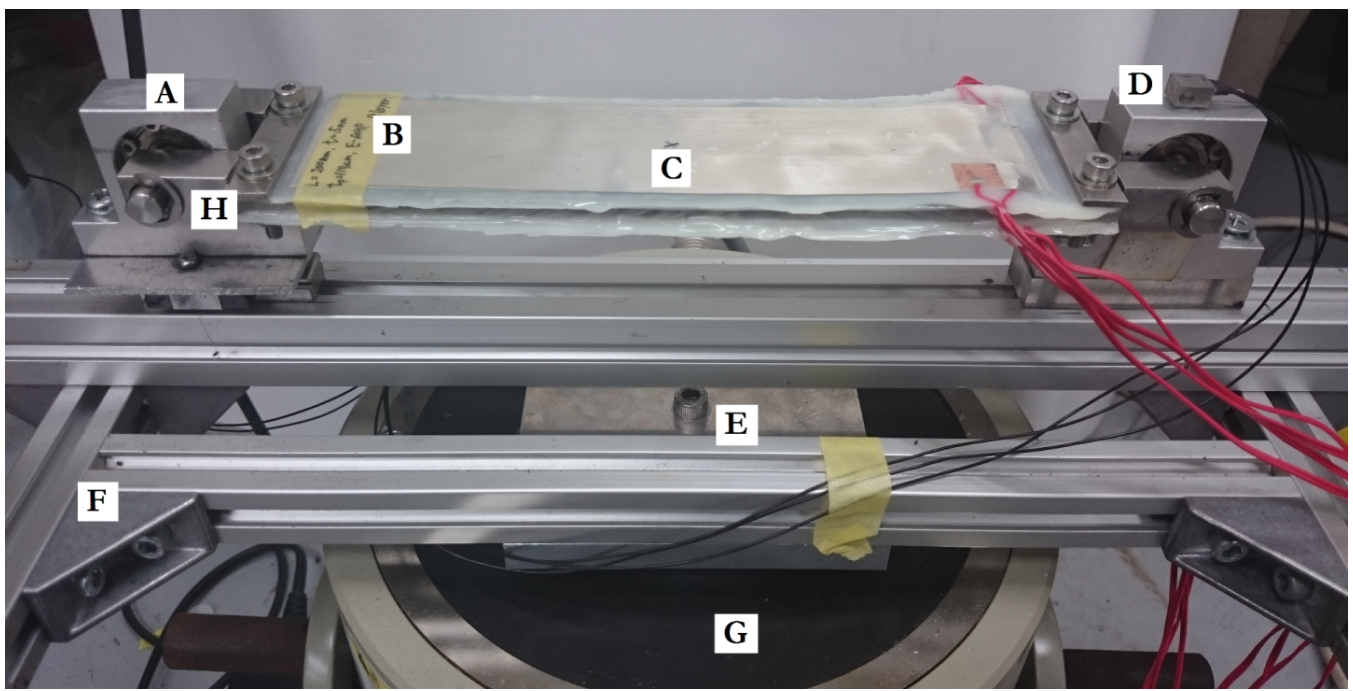

Figure 6. Test-rig used during experiments. (A) bearing housing, $(B)$ multi-layered device, $(C)$ displacement measurement point, $(D)$ accelerometer location, $(E)$ connection of test rig to shaker, $(F)$ test-rig frame, $(G)$ shaker and $(H)$ connector element.

following sections, good agreement between theory and experiment is obtained in regards to the resonant frequency for various simply-supported devices.

Harmonic forcing is applied to the rig using a EMIC Corp shaker (model no. 513-B) with the sinusoidal signal provided by a NF WF1945 multifunction synthesizer via a EMIC Corp power amplifier (model no. 371-A). Displacement measurements at the device mid-span are taken using a IL-300 Keyence Laser Sensor and acceleration levels on the bearing housing, i.e. base accelerations, are measured using a NP-2506 piezoelectric accelerometer. Data acquisition for displacements, accelerations and open circuit voltages is preformed through a 7-channel Keyence NR-500 Data Logger. As a result of non-linearity, whilst obtaining frequency response graphs, it is important to ensure the acceleration level applied across the frequency range remains constant. As no feedback control system was available, at each frequency, a manual tuning of the acceleration level was performed to within $\pm 1 \%$ of the desired level.

\section{Device with $n=2$}

The experimental data obtained for a device where $n=2$ (i.e. 1 piezoelectric layer on either side of the core substrate layer), superimposed with theoretical trends, is provided in Figure 7. The device was subjected to acceleration levels of $2.5 \mathrm{~ms}^{-2}, 5 \mathrm{~ms}^{-2}, 10 \mathrm{~ms}^{-2}, 15 \mathrm{~ms}^{-2}$ and $20 \mathrm{~ms}^{-2}$ with displacement measured at the mid-span and voltage measured across a $1 \mathrm{M} \Omega$ resistor. Note that in this case testing was performed with device layers in series connection. Curvefitting was used to obtain: (i) the mechanical damping ratio, $\gamma$, at each acceleration level; and, (ii) the material non-linear coefficients found in Equations (19)-(20), i.e. $\mu_{s 1}, \mu_{s 2}, \mu_{p 1}$ and $\mu_{p 2}$. For the material combination used in this design it is reasonable to assume that the piezoelectric material will be the greatest contributor to material non-linearity. Therefore in the proceeding analysis it is assumed that $\mu_{s 1}=\mu_{s 2}=0$. Furthermore, over the tested acceleration range, it was found that one non-linear coefficient alone was sufficient to predict the behaviour of the response at differing acceleration levels, i.e. $\mu_{p 2}=0$.

During theoretical calculation, $2.5 \times 10^{14} \mathrm{~Pa}$ was found to be an appropriate estimation for $\mu_{p 1}$, see Figure 7 . Additionally, the following mechanical damping ratios were extracted for increasing acceleration levels: $0.0135,0.0145$, $0.0125,0.015$ and 0.0185 . From the numerical information, an increase in $\gamma$ with acceleration is seen for excitation levels over $10 \mathrm{~ms}^{-2}$; this is expected as detailed in Yao et al. (2004). For lower acceleration levels, where beam energy and 

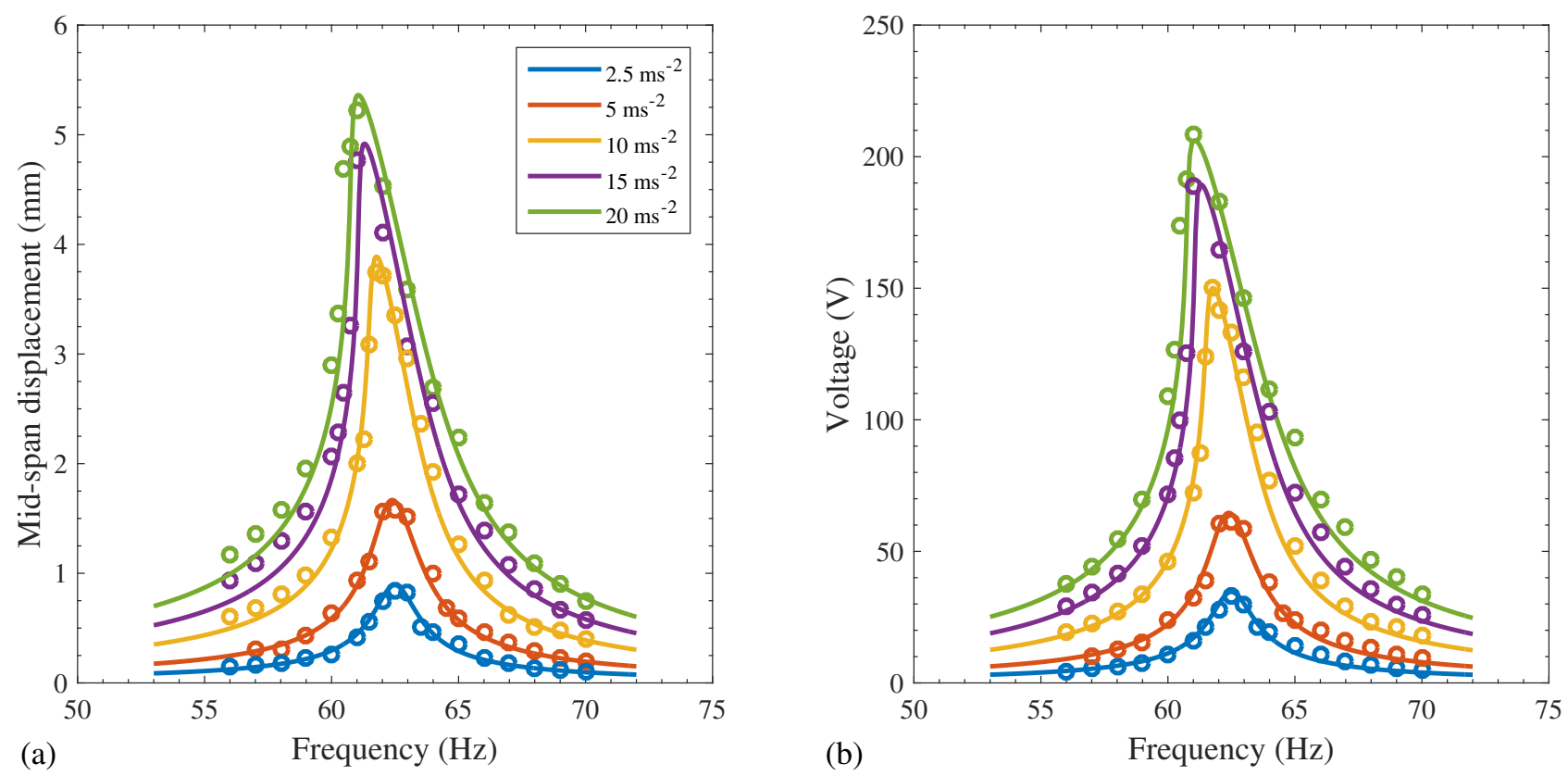

Figure 7. Experimental-theoretical comparisons for a device where $n=2$ at various acceleration levels. All layers are connected in series. Solid lines represent simulated results and markers represent experimental data. (a) Midspan displacement and (b) Voltage.

beam oscillations are small, the friction in the bearing housing and slider is thought to be responsible for the relatively larger measured $\gamma$ values. In terms of experimental-theoretical comparisons, resonant frequencies along with displacement and voltage peaks across the acceleration range are in good agreement. Numerically, the differences in displacement peak magnitude, voltage peak magnitude and resonant frequency at an acceleration level of $20 \mathrm{~ms}^{-2}$, are $2.7 \%, 1.0 \%$ and $0.02 \%$, respectively. The main reason for discrepancies is due to uncertainties in the experiment, particularly with the hand-manufacture of devices; inevitably this will result in deviation from a model which assumes perfect and repeatable manufacture. Other differences arise from the setup of the test rig as the realisation of a beam under perfectly simplysupported conditions is challenging to achieve. Regarding peak voltage, subtle difference may also occur as a result of variability in the magnitude of $\mathrm{d}_{31}$, which the PVDF manufacture quotes between $20 \mathrm{pC} / \mathrm{N}$ and $30 \mathrm{pC} / \mathrm{N}$.

\section{Device with $n=6$}

The experimental data and theoretical results for a device where $n=6$ are provided in Figure 8 . The device was subjected to the same acceleration levels and load resistance to those quoted in the previous section, however, here, layers are connected in parallel to demonstrate further capabilities of the model. Note, whilst obtaining theoretical trends, the previously found $\mu_{p 1}$ of $2.5 \times 10^{14} \mathrm{~Pa}$ is used.

Figure 8 shows good agreement between theoretical trends and the experimental data. The mechanical damping ratios for increasing levels of acceleration are calculated to be 0.01, 0.0105, 0.01, 0.0108 and 0.0118. Experimental-theoretical percentage differences at $20 \mathrm{~ms}^{-2}$ for displacement peak magnitude, voltage peak magnitude and resonant frequency are found to be $2.7 \%, 1.0 \%$ and $0.02 \%$, respectively, with differences resulting from aforementioned reasoning in the "Device with $n=2$ " section. 

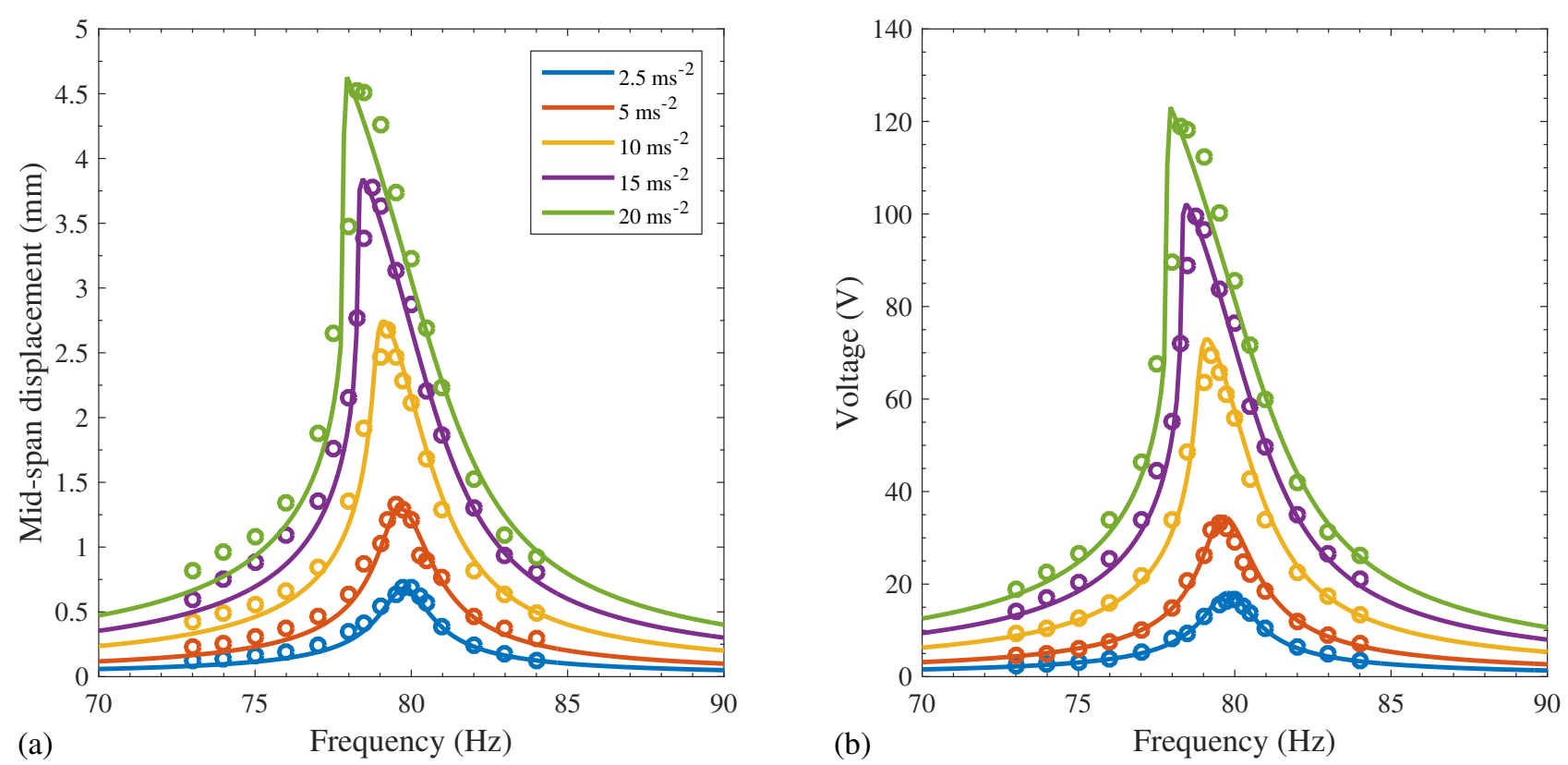

Figure 8. Experimental-theoretical comparisons for a device where $n=6$ at various acceleration levels. All layers are connected in parallel. Solid lines represent simulated results and markers represent experimental data. (a) Midspan displacement and (b) Voltage.

\section{Device with $n=8$}

A device where $n=8$ was also manufactured and tested, with Figures 9 and 10 providing experimental data and theoretical trends for the layers in series and parallel connection, respectively. During theoretical simulation, the previously obtained $\mu_{p 1}$ magnitude of $2.5 \times 10^{14} \mathrm{~Pa}$ was again utilised, with experimental-theoretical comparisons showing good agreement. At an acceleration level of $20 \mathrm{~ms}^{-2}$, differences in peak displacement, peak voltage and resonant frequency, whilst layers are in series connection, are $2.2 \%, 4.7 \%$ and $0.7 \%$, respectively. Similarly, for layers in parallel connection, difference of $4.2 \%, 2.5 \%$ and $0.7 \%$ are obtained. The extracted mechanical damping ratios for increasing acceleration levels were $0.0145,0.0115,0.0105,0.0115$ and 0.0122 ; a trend similar to that observed for devices with $n=2$ and $n=6$.

The results in Figures 7-10 indicate that the theoretical model is fully capable of predicting dynamic behaviour and performance for a range of simply-supported multi-layered devices with various electrical connection between layers. At an acceleration of $20 \mathrm{~ms}^{-2}$, percentage differences in all cases were found to be below $1 \%$ for resonant frequencies, and below $5 \%$ for peak displacements and peak voltages. Note how a direct comparison on the performance of the above devices cannot be made due to differing lengths of piezoelectric material and differing resonant frequencies; an unbiased performance comparison study is presented in the "Multi-layered devices" section.

It is evident from the presented results that for simply-supported devices, material non-linearity is dominant over geometric non-linearity. Such dominance gives rise to a softening phenomenon whereby increasing acceleration levels cause reductions in the resonant frequency of devices; this observation was also made in previous work on cantilevered piezoelectric energy harvesters, see Stanton et al. (2012) and Patel et al. (2016). In the experiments conducted here, resonant frequency shifts between acceleration levels of $2.5 \mathrm{~ms}^{-2}$ and $20 \mathrm{~ms}^{-2}$, were found to be $2.4 \%, 1.9 \%$ and $1.7 \%$ for the $n=2, n=6$ and $n=8$ devices, respectively. The reduced sensitivity with increasing $n$ can be attributed to the reduced peak deflections resulting from an increased stiffness. Although frequency shifts for the presented cases are low, different device composition, form and dimension will affect the extent of non-linearity; as an example, manufacturing the device from silicon rubber and PVDF greatly increases the level of non-linearity as shown in Patel et al. (2016). Therefore it 

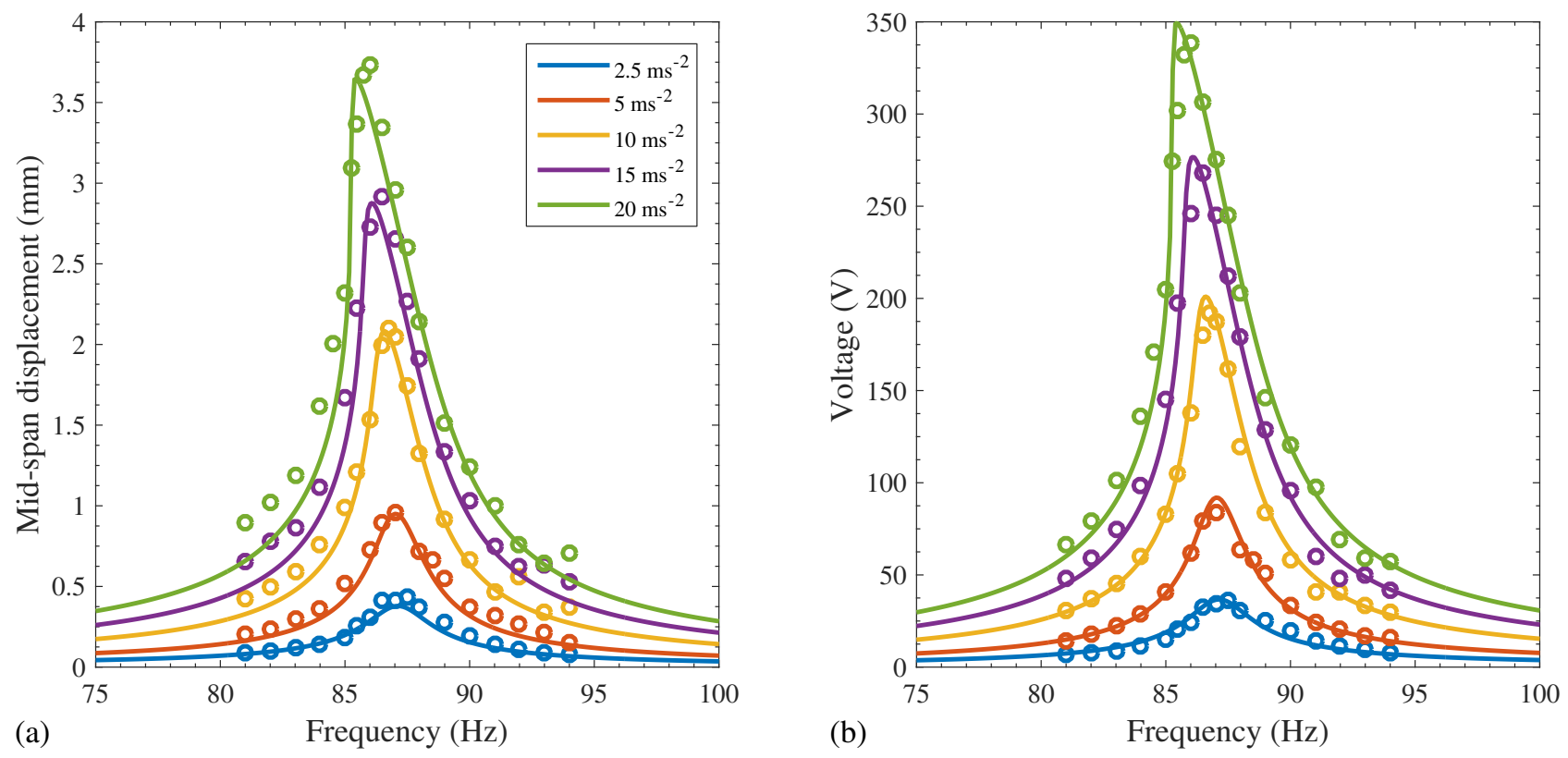

Figure 9. Experimental-theoretical comparisons for a device where $n=8$ at various acceleration levels. All layers are connected in series. Solid lines represent simulated results and markers represent experimental data. (a) Midspan displacement and (b) Voltage.
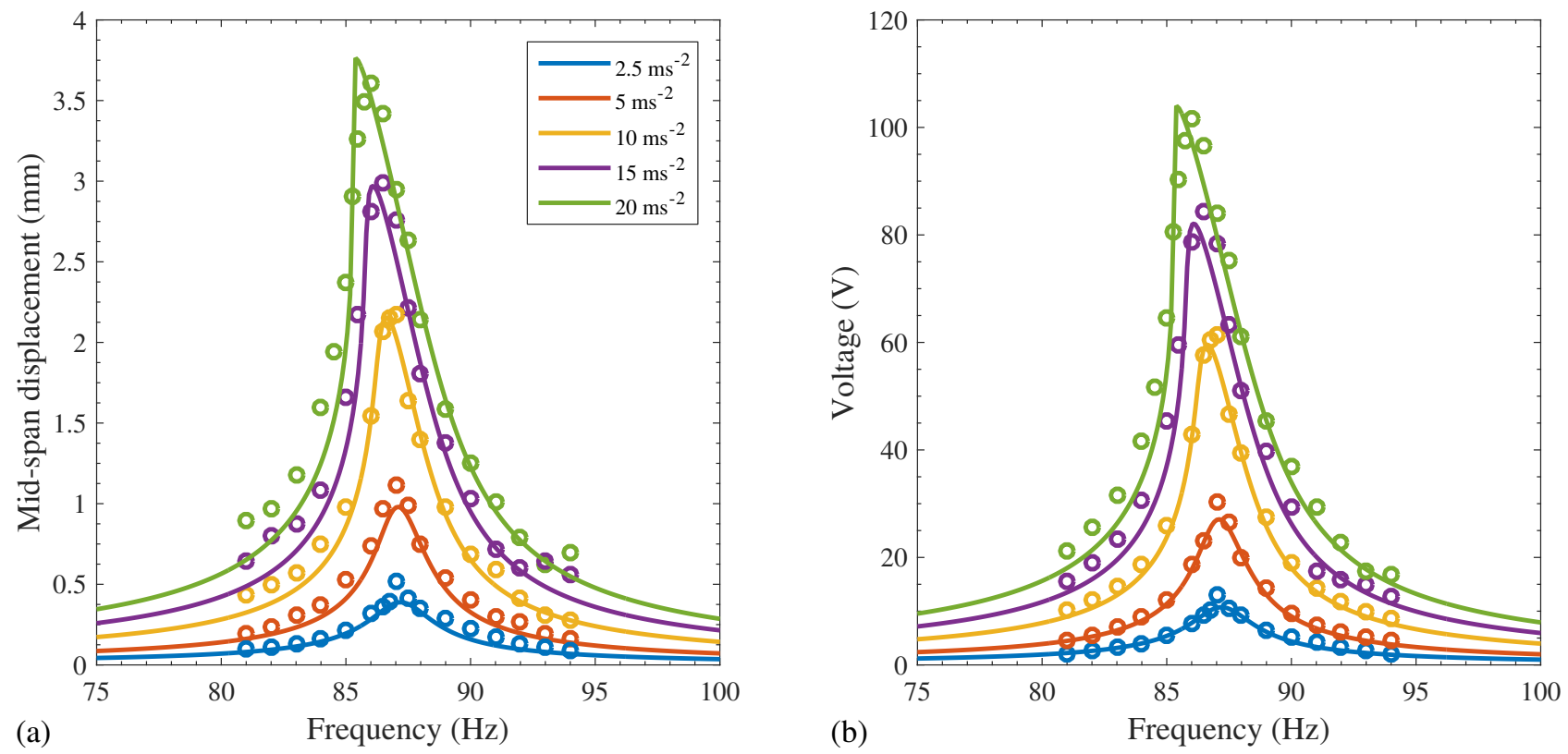

Figure 10. Experimental-theoretical comparisons for a device where $n=8$ at various acceleration levels. All layers are connected in parallel. Solid lines represent simulated results and markers represent experimental data. (a) Midspan displacement and (b) Voltage.

was still important to include non-linear material and geometric modelling for model versatility and potential future model use. Moreover, the importance of including and understanding the effects of non-linearity are essential to effectively design an energy harvester for a target application. Energy harvesters are designed such that their resonant frequency matches the dominant excitation frequency. Without knowledge of frequency shifts due to non-linearity, in a predominantly $20 \mathrm{~ms}^{-2}$ 
environment, the device with $n=2$ would inadvertently be designed to operate $21 \%$ less efficiently, i.e. designed assuming the device resonance would be $62.5 \mathrm{~Hz}$ at $20 \mathrm{~ms}^{-2}$, providing a voltage of $162.9 \mathrm{~V}$, as opposed to the actual resonance of $61 \mathrm{~Hz}$, where a potential $206.1 \mathrm{~V}$ can be harvested.

\section{Model Utilisation}

In this section the developed model is used to investigate the influence of using different boundary conditions and the impact of stacking piezoelectric layers.

\section{Investigation of using different boundary conditions}

Piezoelectric energy harvesters are often designed and manufactured for application as cantilevered devices. The simplicity in manufacture, the low resonant frequencies and the large deflections are all factors which make a cantilevered piezoelectric energy harvester appealing. However, various alternative boundary conditions are possible and may have been overlooked in terms of their energy harvesting potential. In this section, a theoretical performance comparison will be presented between a conventional 2-layered device, see Table 1 for dimensions and material properties, in a simplysupported condition and cantilevered condition. For the simply-supported case the device is as depicted in Figure 1. However, for the cantilevered case the right-side steel connection element is removed as, unfavourably, this would have acted as a tip mass increasing the difference in natural frequency between simply-supported and cantilevered setups.

Although Equations (30) and (31) are common to both boundary conditions, differences in modelling the two systems are found in the extraction of natural frequencies and mode shapes. As mentioned in the "Transfer matrices with axial force" section, the matrix providing $\omega_{r}$ for a simply-supported device is $\left[\mathbf{U}_{\text {overall }}(1,2) \mathbf{U}_{\text {overall }}(1,4) ; \mathbf{U}_{\text {overall }}(3,2) \mathbf{U}_{\text {overall }}(3,4)\right]$. In contrast, for a cantilevered device the deflection and slope at the clamped end are zero, along with the moment and shear force at the free end. This leaves the $2 \times 2$ matrix $\left[\mathbf{U}_{\text {overall }}(3,3) \mathbf{U}_{\text {overall }}(3,4) ; \mathbf{U}_{\text {overall }}(4,3) \mathbf{U}_{\text {overall }}(4,4)\right]$, whose zero determinant will provide the natural frequency from which mode shape is then obtained. Therefore, distinction between cantilevered and simply-supported devices arises from modelling changes to both the natural frequency and mode shape of the system. Figure 11 shows the maximum displacement and voltage generated by the simply-supported and cantilevered device at an acceleration level of $10 \mathrm{~ms}^{-2}$ connected to a $1 \mathrm{M} \Omega$ resistor in linear conditions.

The results show both a large reduction in resonant frequency and an increase in maximum deflection through the use of a clamped-free boundary condition. However, for this to be an unbiased design comparison, when analysing the performance of devices, natural frequencies should be identical. This logic allows both devices to be used in the same application; more details on this methodology can be found in Patel (2012). To this end, the core substrate (PET) layer thickness, $t_{s}$, of the cantilevered device is increased to $7.54 \mathrm{~mm}$ and the response, along with that of a simply-supported device, provided in Figure 12. Note the use of substrate thickness for frequency control as opposed to say device length or piezoelectric layer thickness. The reasoning behind this is twofold: (i) to ensure the volume of piezoelectric material in devices is the same and (ii) to not alter the basic footprint of devices.

Now that the resonant frequencies are matched, the displacement of both devices (note an equal mechanical damping ratio is assumed) is almost identical. However, the voltage generated by the device in simply-supported conditions is more than double that generated by an equivalent device in cantilevered boundary conditions. Numerically, a maximum of $141.8 \mathrm{~V}$ and $65.5 \mathrm{~V}$ is generated for the simply-supported device and the cantilevered device respectively, i.e. a $116 \%$ increase. In terms of power generated by devices, the simply-supported device is superior by approximately $370 \%$.

Figure 13 shows the strain distribution along the device for both cases considered and provides insight into the observed behaviour. Despite the fact that the cantilevered device has a thicker substrate layer, thereby increasing the distance 

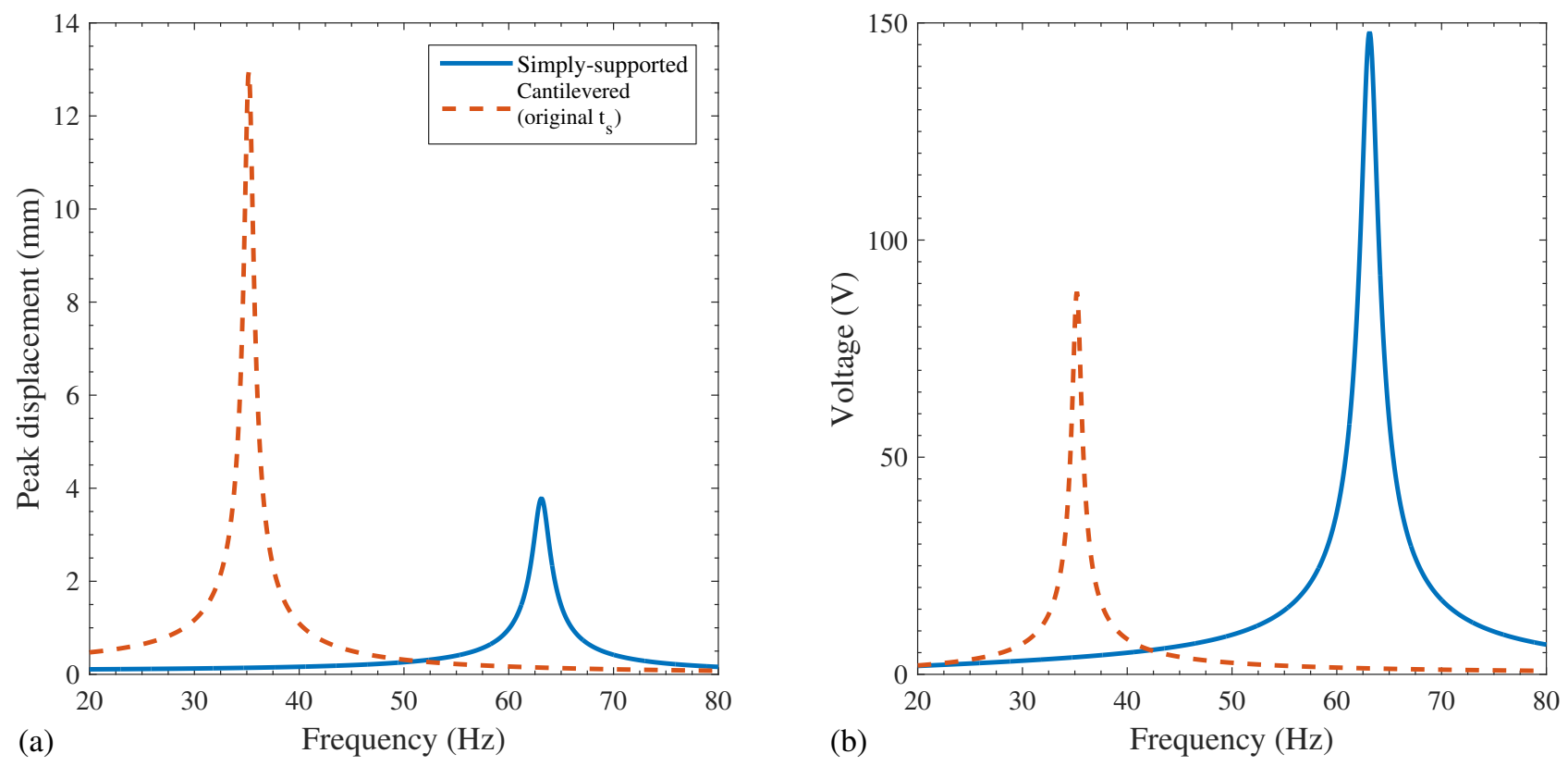

Figure 11. Theoretical frequency responses for: (a) peak displacement; and, (b) voltage, whilst comparing different boundary conditions. For simply-supported conditions, the peak displacement measurement is taken at $L / 2$, whereas for cantilevered conditions the measurement is at $L$. The centre substrate thickness, $t_{s}$, is the same for both devices.
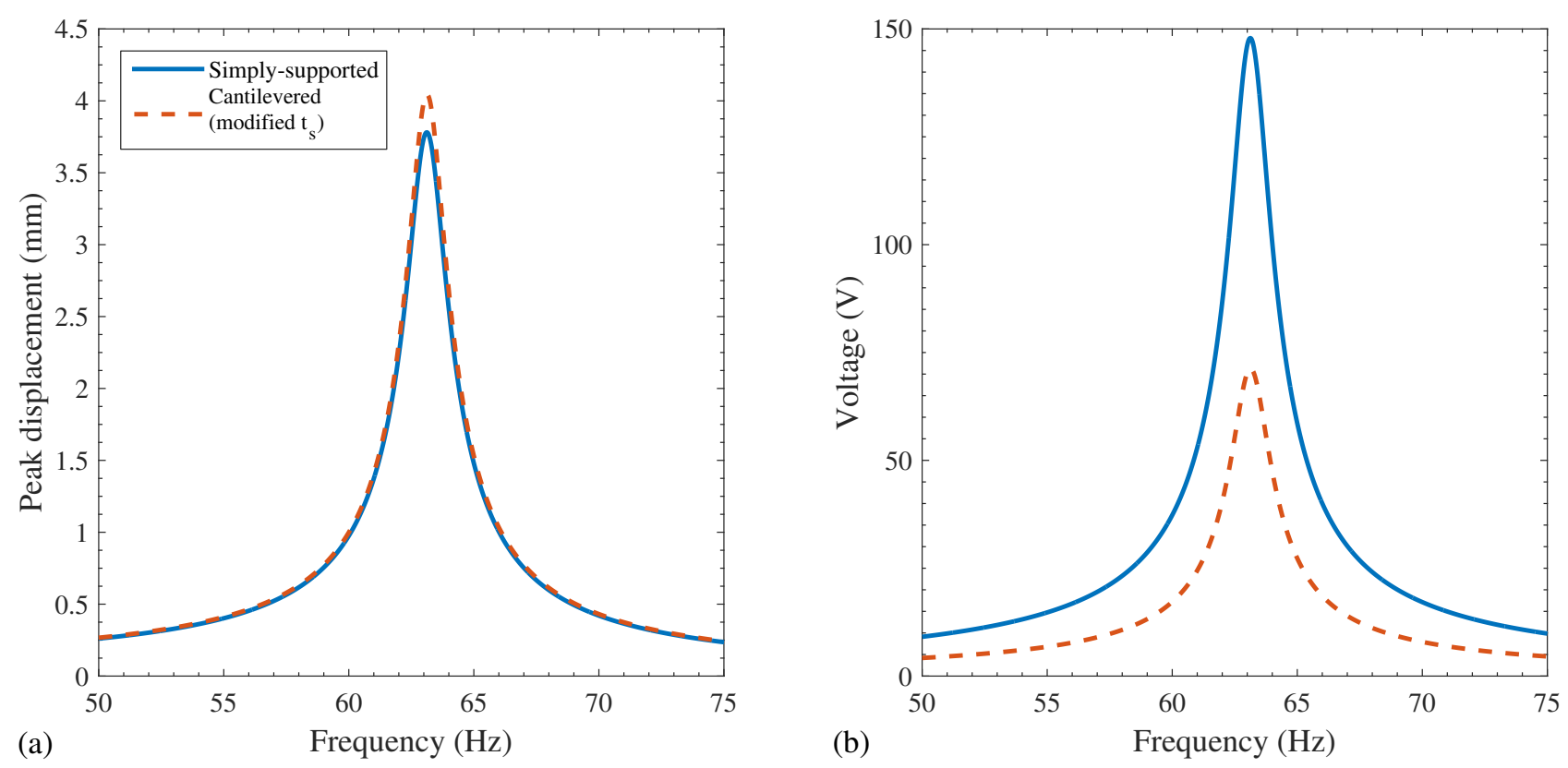

Figure 12. Theoretical frequency responses for: (a) peak displacement; and, (b) voltage, whilst comparing different boundary conditions. For simply-supported conditions, the peak displacement measurement is taken at $L / 2$, whereas for cantilevered conditions the measurement is at $L . t_{s}$ used to adjust the resonant frequency of the cantilevered device.

between neutral axis and piezoelectric material centre axis $(3.88 \mathrm{~mm}$ compared to $2.065 \mathrm{~mm})$, the strain levels present in the piezoelectric layer in a simply-supported design are superior. Also, importantly, the distribution of strain is more uniform along the device length creating a more favourable energy harvesting system. 


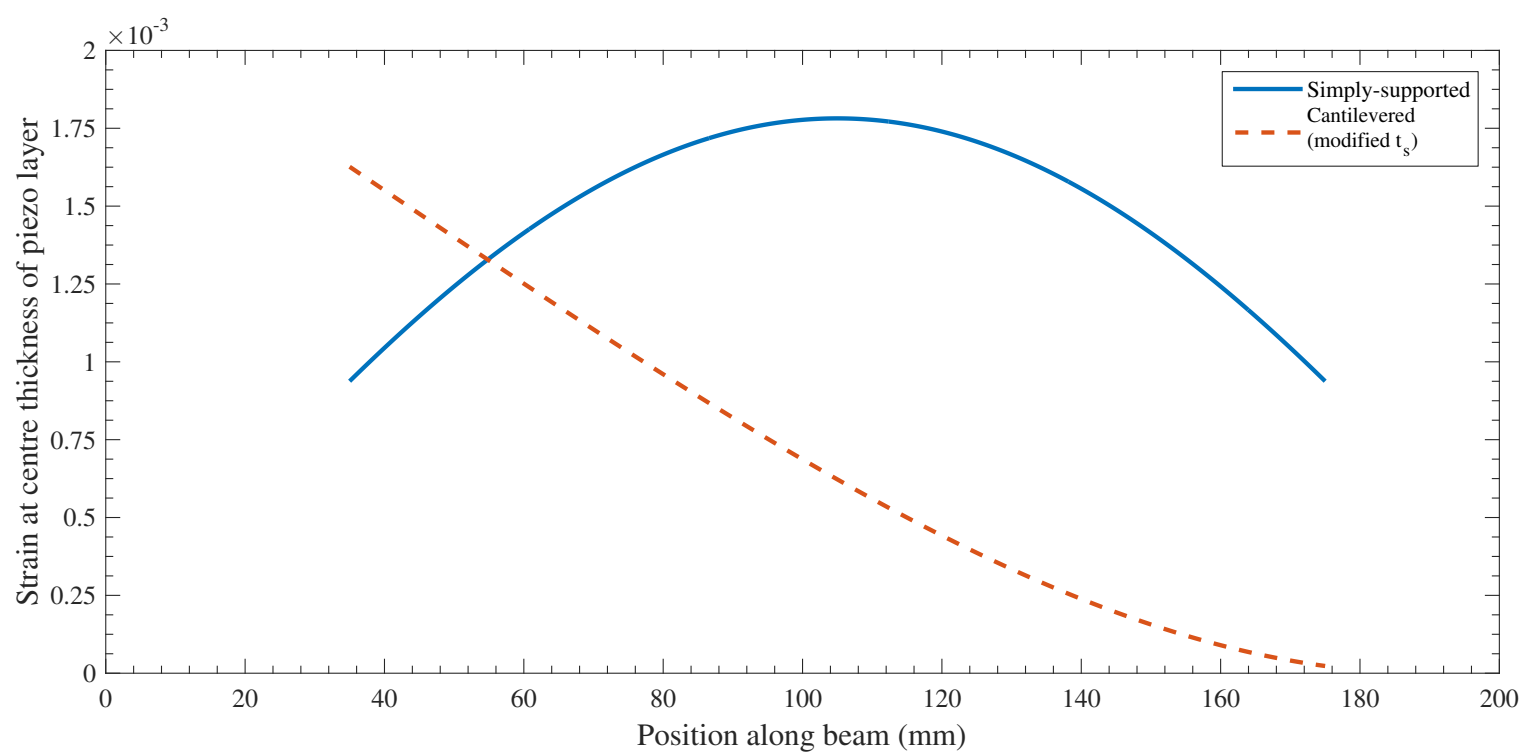

Figure 13. Theoretical strain distribution at the centre of the piezoelectric layer for various boundary conditions. Distance between the piezoelectric material centre axis and neutral axis for the simply-supported and cantilevered device are $2.065 \mathrm{~mm}$ and $3.88 \mathrm{~mm}$, respectively.

If the higher fundamental frequencies of simply-supported devices are a concern then manufacture with a thinner core substrate layer is an option, and as the example has illustrated, the performance still outweighs that of a cantilevered device. If however manufacture with a thinner core substrate layer is impracticable then an axial compressive pre-load, applied to the device at its supports, can be used to lower the natural frequency.

\section{Multi-layered devices}

Using substrate thickness for $\omega_{n}$ control In this section the performances of devices are theoretically compared whilst altering the number of layers, $n$. The logic behind increasing the number of layers as opposed to using a single piezoelectric layer with increased thickness is to create a more robust device. By increasing the number of piezoelectric layers, overall performance of the device is less affected if one piezoelectric layer were to become damaged. Devices comprise of PET as the core substrate, with $0.11 \mathrm{~mm}$ PVDF and $0.1 \mathrm{~mm}$ PET making up the stacking layers. Section widths remain constant throughout the study with numerical information provided in Table 1 . As $n$ is increased, naturally, the fundamental frequency will change, creating a situation of unbiased design comparison. The conventional design, i.e. $n=2$, with a substrate thickness of $4 \mathrm{~mm}$, is used to obtain a target $\omega_{1}$ of $62.5 \mathrm{~Hz}$. The fundamental frequency of designs with $n>2$ are therefore tuned to $62.5 \mathrm{~Hz}$ by selecting an appropriate thickness for the core substrate layer. All designs are simplysupported, see Figure 1, subjected to a $10 \mathrm{~ms}^{-2}$ base acceleration, and assumed to have an identical 0.0125 mechanical damping ratio and zero adhesive thickness. Figure 14 provides the peak power at various load resistances when devices with $n=2, n=6$ and $n=10$ have their layers in either a series, parallel or series-parallel connection, see the "Multilayered device models" section. For each load resistance and electrical connection a sweep of the excitation frequency between $\pm 15 \%$ of $\omega_{1}$ is performed to determine the peak voltage, hence peak power, in those conditions.

Figure 14 shows that the peak power from a device increases with $n$ and is independent of electrical connection. The electrical connection between layers can however be used to control the load resistance at which this peak power occurs. For example, for a device with $n=10$ this resistance can either be $0.18 \mathrm{M} \Omega, 4.6 \mathrm{M} \Omega$ or $18 \mathrm{M} \Omega$. For a device with $n=2$, 


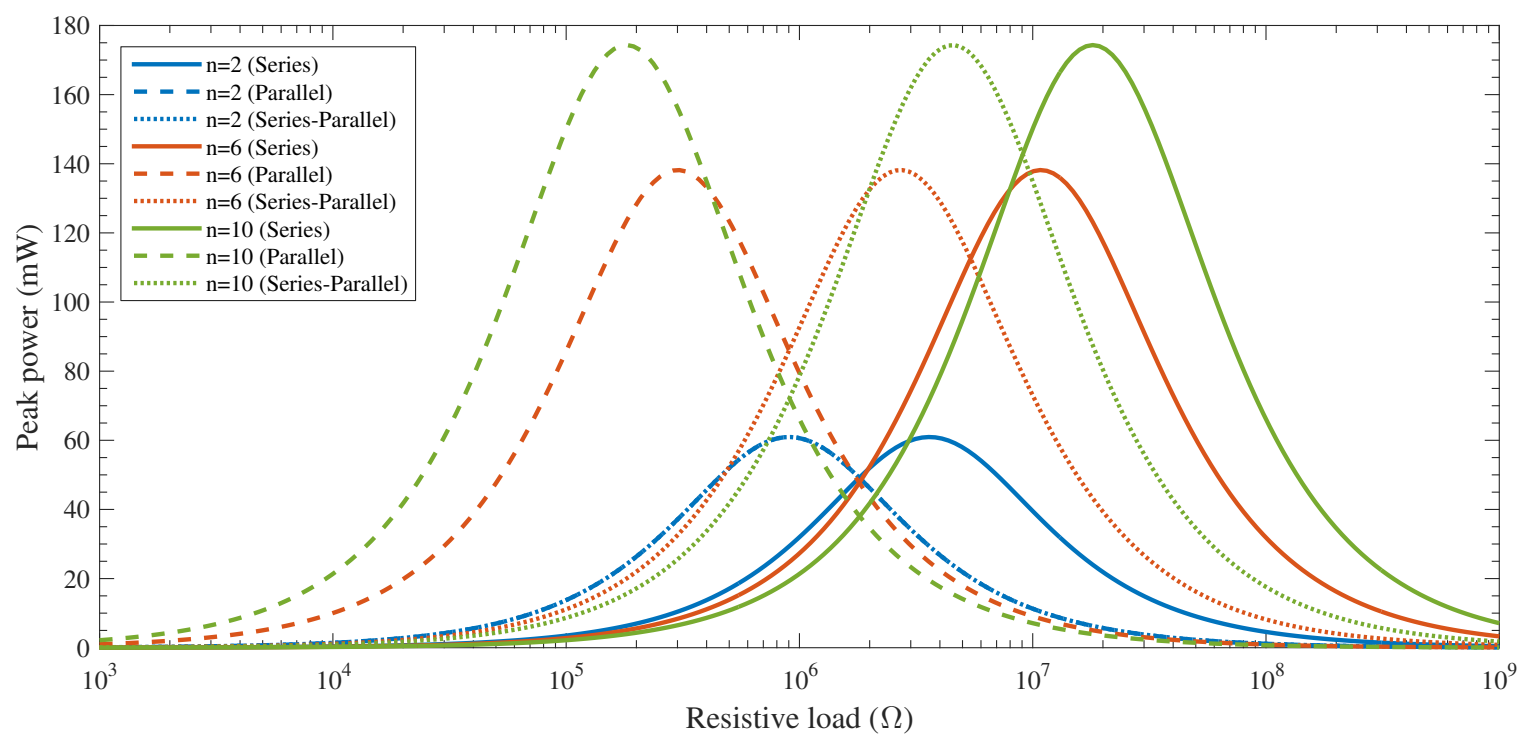

Figure 14. Theoretical plots of peak power vs load resistance for devices with differing $n$. All devices are simulated with various electrical connection between layers. Note, when $n=2$, the parallel and series-parallel powers are identical, giving superimposed lines.

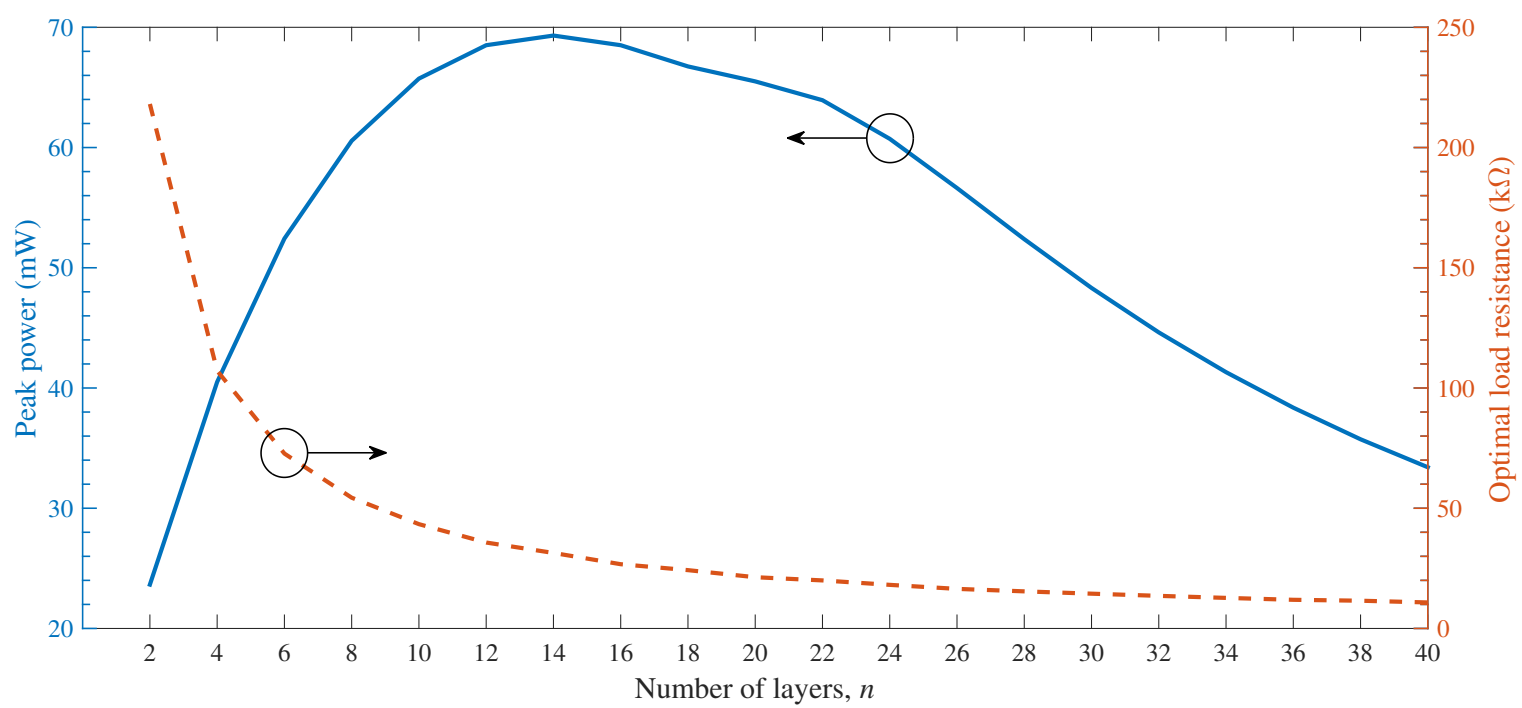

Figure 15. Theoretical relationship between number of layers, peak power and optimum load resistance when layers are connected in parallel. Note: scale on vertical axis for the power is $20-30 \mathrm{~mW}$.

the load resistance providing peak power for a parallel and series-parallel connection is the same; this observation can also be deduced by examining the circuit diagrams in Figures 4 and 5 .

To make this a more comprehensive study on the performance of multi-layered devices, a greater range of $n$ was simulated. Figure 15 shows trends between $n$, the peak power and the corresponding optimum resistance. Note that $n>20$, whilst using the core substrate thickness for natural frequency correction, is not feasible in this case; the core substrate thickness is already as low as $0.279 \mathrm{~mm}$ when $n=20$. It has been established that electrical connection does not affect peak power so subsequent simulations are performed with device layers in parallel connection only. 


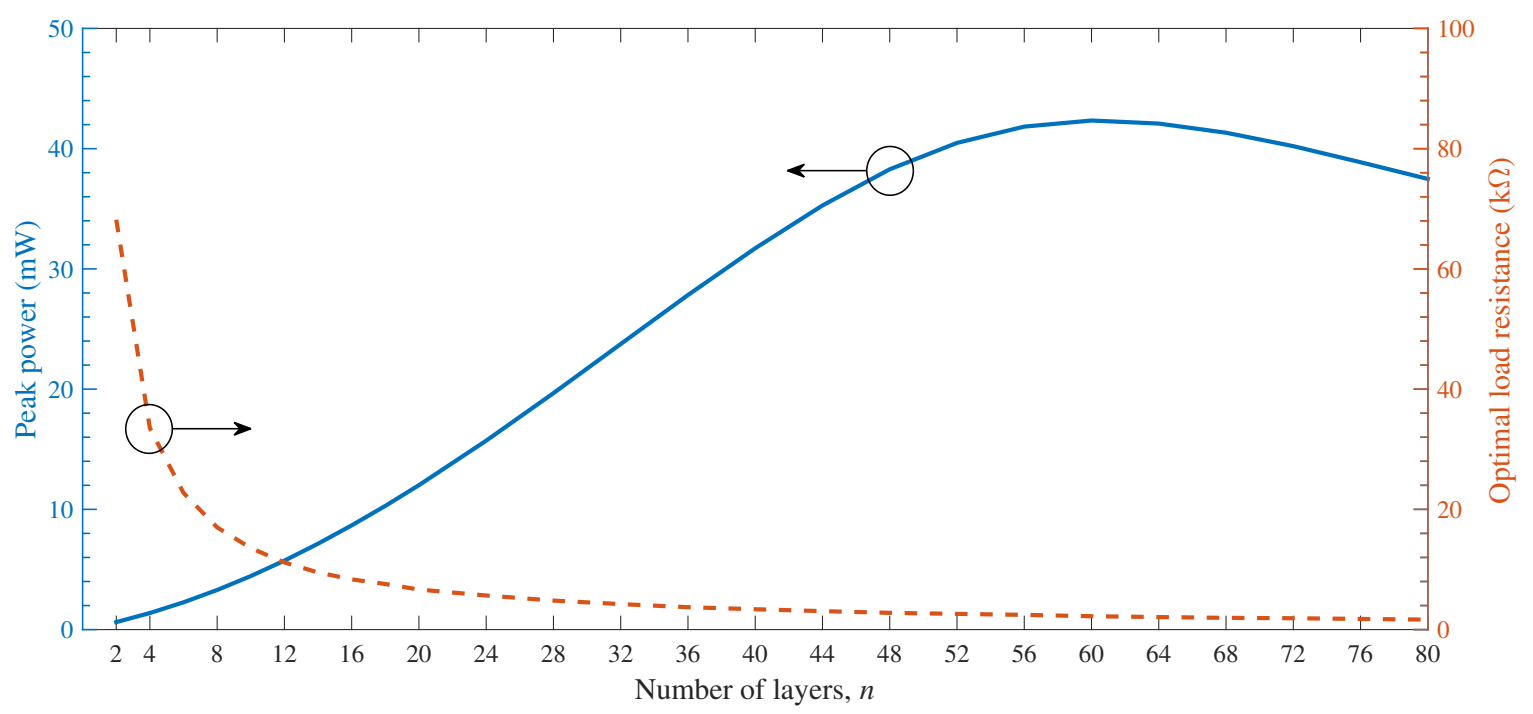

Figure 16. Theoretical relationship between number of layers, peak power and optimum load resistance when layers are connected in parallel. A stiffer core substrate is used in this example.

Figure 15 shows that there is an optimum $n$ value providing maximum performance, and for a device of this composition and base dimension, it is approximately $70 \mathrm{~mW}$ when $n=12$. As $n$ is increased the volume of piezoelectric material increases and so one would expect superior performance. However, a balance between number of layers and maximum power is clear and this results from: (i) reductions in the core substrate thickness whilst increasing $n$, causing decreased distance between the neutral axis and the piezoelectric material; and, (ii) the decrease in maximum displacement, both of which reduce strain in the piezoelectric layers. Further inspection of Figure 15 indicates that one can be mislead by the optimum $n$ value from such parameter studies; comparing a 12-layer device to one with 2-layers, an approximate tripling in performance is observed, although six times more piezoelectric material is required. If space restriction is not a factor, in this example it is better to use several 2-layer devices. However, if space is limited then the 12-layer device is recommended as it has a similar overall volume to the 2-layer device but performs substantially better.

Results from a second simulation on multi-layered devices are provided in Figure 16. In this example a stiffer material is used for the core substrate layer (Al: $E=69 \mathrm{GPa}$ and $\rho=2700 \mathrm{GPa}$ ). Stacking layer materials and base dimensions remain identical to the previous example. The natural frequency of the conventional 2-layered design in this case is $201 \mathrm{~Hz}$, in comparison to $62.5 \mathrm{~Hz}$ when the core substrate was PET. The core substrate thickness is again modified and calculated for each design so that all devices possess a natural frequency of $201 \mathrm{~Hz}$.

Figure 16 shows a similar general trend between $n$ and peak power to that obtained in Figure 15. However, the optimum $n$ has now increased to 60 . A stiffer core substrate allows for smaller reductions in substrate thickness with increasing $n$ so a favourable distance between neutral axis and piezoelectric material is maintained. This also translates to benefits in terms of power-per-unit-volume of piezoelectric material. Numerically, a device with 12-layers outperforms a conventional 2layer device by a factor of eight, although the piezoelectric material increase is only a factor of six. Comparing the 2-layer device to a 60-layer device, a 30 times increase in material yields a 68 times increase in power. Note that due to advances in the additive manufacturing of piezoelectric materials and devices (Lee and Tarbutton (2014)), it is thought that the production of devices with large numbers of layers will be a feasible option in the near future.

Significance of frequency control It is important to maintain a constant natural frequency across the range of devices being compared as failure to do so is likely to cause biased conclusions regarding optimum designs as indicated in Figure 17. It can be seen that disregarding natural frequency control results in a design with $n>80$ providing peak 


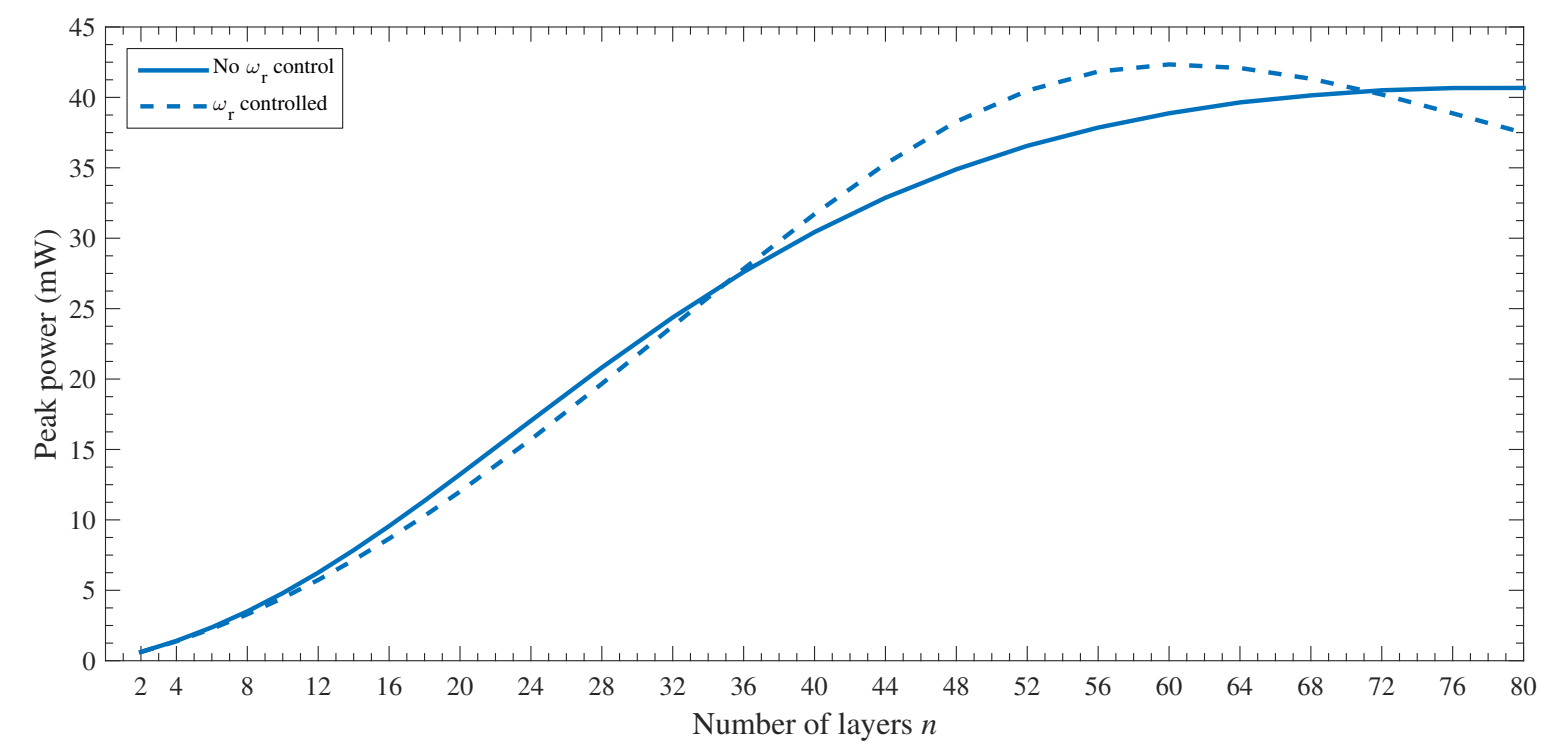

Figure 17. Theoretical relationship between number of layers and peak power whilst either correcting for natural frequencies changes, unbiased design comparisons, or not, biased design comparisons.

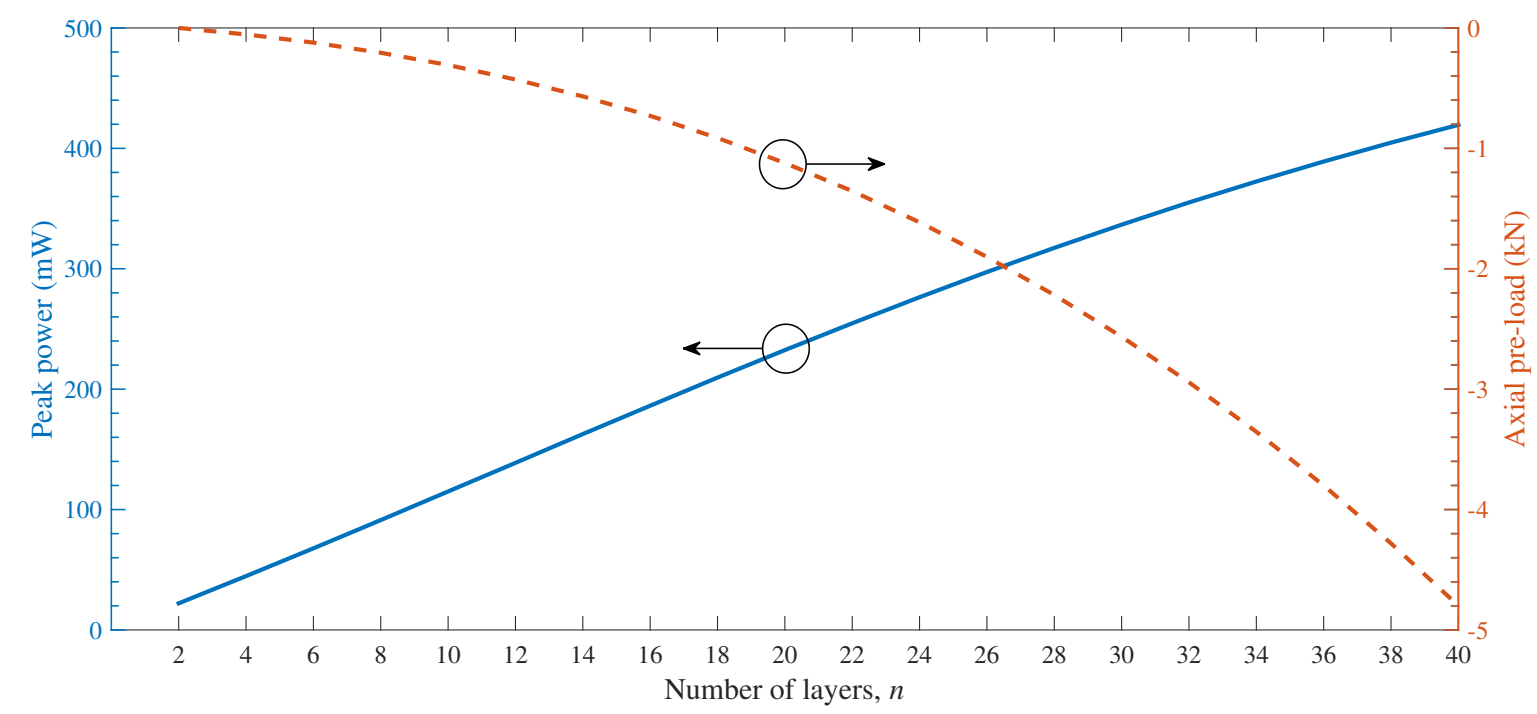

Figure 18. Theoretical relationship between number of layers, peak power and required compression for natural frequency control when layers are connected in parallel.

power as opposed to the estimation of $n=60$ during the earlier fairer comparison study. This conclusion is drawn from designs with a relatively stiff core substrate layer, and the issue will only be amplified for cases such as that of the original design (core substrate layer of PET) where fundamental frequency changes are more sensitive to increases in the number of layers.

Using axial pre-load for $\omega_{r}$ control In this section, axial pre-load is used as the natural frequency control mechanism as opposed to the substrate thickness in previous sections. The device composition and core dimensions are those provided in Table 1 for a 2-layer device. The addition of layers increases the natural frequency of a device and therefore compressive pre-load is applied to devices of $n>2$. 
Figure 18 shows a different trend for peak power vs. $n$ to that obtained in Figure 15 for a comparable study and highlights the significance of the frequency control method chosen. Across the examined range, whilst using compression for natural frequency control, the power is seen to continually increase with the number of layers. As opposed to the study when substrate thickness was used for frequency control, the distance between the piezoelectric material and neutral axis now remains constant and so detrimental effects from reducing this distance are no longer present. However, in terms of power-per-volume of piezoelectric material, performance of a 2-layer device is on-par with that of the 40-layer device.

Since axial compression is utilised, it is important to ensure the beam will not buckle through the application of this pre-load. The Euler buckling formula for a simply-supported column is used to estimate the load to buckling:

$$
T_{c r}=\frac{(E I) \pi^{2}}{L^{2}}
$$

where $T_{c r}$ is the critical compression force, $E I$ is the flexural rigidity and $L$ is the beam length. Since the device under examination has several sections, for simplification, only the multi-layered section is considered in the above formula. This is a reasonable assumption as the multi-layered section is the longest, and across the majority of $n$ values has a small $(E I)$ magnitude. Taking the case when $n=40$, using Equation (33) results in a $T_{c r}$ of $\sim 58 \mathrm{kN}$. This is an order of magnitude greater than the $4.8 \mathrm{kN}$, see Figure 18, required for natural frequency rectification for a 40-layer device. Therefore it can be concluded that the beam will not buckle and the application of compression is an acceptable means for natural frequency control in this configuration.

\section{Conclusion}

This paper has presented the development, validation and utilisation of an analytical non-linear model for multi-layered energy harvesters. Both geometric and material non-linearity were considered however it was seen from testing that material non-linearity is the dominant contributor in simply-supported harvesters, causing resonance softening with increasing excitation levels. The model was formulated to also include axial pre-loading which was exploited as a mechanism for frequency control in a presented parametric study.

Model validation using experimental data from several multi-layered simply-supported beam energy harvesters was provided. The model was shown to be capable of predicting the frequency response for a 2- 6- and 8-layered device, regardless of electrical connection between layers, each excited at base acceleration levels between $2.5 \mathrm{~ms}^{-2}$ and $20 \mathrm{~ms}^{-2}$. It was found that for this particular acceleration range and device composition, steel, PET and PVDF, only one material non-linear coefficient was required: $\mu_{p 1}=2.5 \times 10^{14} \mathrm{~Pa}$ for the PVDF film. For all devices, theoretical-experimental percentage differences in resonant frequency, mid-span displacement and peak voltage were below 5\%, and this is mostly attributed to the manufacturing uncertainties.

The analytical model was used to compare the performance of devices in simply-supported and cantilevered beam boundary conditions. For unbiased comparisons, and in keeping the device footprint and piezoelectric material volume constant, the substrate thickness was used to match the natural frequency of both devices. The outcome indicated that simply-supported devices are superior, providing a $116 \%$ increase in peak voltage at the same frequency due to an improved strain distribution along device length. The example demonstrated simply-supported devices can be manufactured with thinner substrate layers, so their natural frequencies are comparable with cantilevers, and remain a more favourable energy harvesting setup.

Parametric studies on multi-layered devices showed that whilst using the core substrate thickness for frequency control, an optimum number of piezoelectric-thin substrate layer stacks, $n$, exist. This results from a balance between (i) increases in piezoelectric material and their position in relation to the neutral axis, and (ii) a reduction in deflection with increasing 
$n$. Opting for a higher number of layers can be advantageous but possible benefits are closely linked to the material composition of the initial 2-layer device. For example, using PET as the core substrate provided no benefit in the powerper-unit-volume of PVDF when increasing the layer number. In contrast, using $\mathrm{Al}$ as the core substrate layer, showed a possible 2.3 times improvement in power-per-unit volume when using a 60-layer device over a conventional 2-layer bimorph. In any case, if space is limited, and an improvement in peak power is required without concern for power-perunit volume of piezoelectric material, then multi-layered devices are a viable option.

\section{Acknowledgements}

RP's research was partially supported by the Short-term Postdoctoral Fellowship for Overseas Researchers program from Japan Society for the Promotion of Science (JSPS).

\section{References}

\section{References}

Bokaian A (1988) Natural frequencies of beams under compressive axial loads. Journal of Sound and Vibration 126: 49-65.

Bokaian A (1990) Natural frequencies of beams under tensile axial loads. Journal of Sound and Vibration 142: 481-498.

Erturk A and Inman DJ (2008) A distribution parameter electromechanical model for cantilever piezoelectric energy harvesters. Journal of Vibration and Acoustics 130: 041002 (15pp).

Erturk A, Tarazaga PA, Farmer JR and Inman DJ (2009) Effect of strain nodes and electrode configuration on piezoelectric energy harvesting from cantilevered beams. Journal of Vibration and Acoustics 131: 011010 (11pp).

Jackson N, OKeeffe R, Waldron F, ONeill M and Mathewson A (2014) Evaluation of low-acceleration mems piezoelectric energy harvesting devices. Microsystem Technologies 20: 671-680.

Lee C and Tarbutton JA (2014) Electric poling-assisted additive manufacturing process for pvdf polymer-based piezoelectric device applications. Smart Materials and Structures 23: 095044 (7pp).

Leland ES and Wright PK (2006) Resonance tuning of piezoelectric vibration energy scavenging generators using compressive axial preload. Smart Materials and Structures 15: 1413-1420.

MIDÉ (2015) MIDÉ - Engineering Smart Technology Products. Website last accessed on 26-06-2015, URL:http: //www. mide.com.

Patel R (2012) Modelling Analysis and Optimisation of Cantilever Piezoelectric Energy Harvesters. PhD Thesis, University of Nottingham, University Park, Nottingham, UK.

Patel R, McWilliam S and Popov AA (2014) Optimization of piezoelectric cantilever energy harvesters including non-linear effects. Smart Materials and Structures 23: 085002 (17pp).

Patel R, Tanaka Y, McWilliam S, Mutsuda H and Popov AA (2016) Model refinements and experimental testing of highly flexible piezoelectric energy harvesters. Journal of Sound and Vibration 368: 87-102.

Pestel EC and Leckie FA (1963) Matrix Methods in Elastomechanics. New York, America: McGraw-Hill.

Stanton SC, Erturk A, Mann BP, Dowell EH and Inman DJ (2012) Nonlinear nonconservative behavior and modeling of piezoelectric energy harvesters including proof mass effects. Journal of Intelligent Material Systems and Structures 23: 183-199.

Xu C, Liang Z, Ren B, Di W, Luo H, Wang D, Wang K and Chen Z (2013) Bi-stable energy harvesting based on a simply supported piezoelectric buckled beam. Journal of Applied Physics 114: 114507 (5pp).

Yao LQ, Zhang JG, Lu L and Lai MO (2004) Nonlinear dynamic characteristics of piezoelectric bending actuators under strong applied electric field. Journal of Microelectomechanical Systems 13: 645-652. 
Zhang L, Oh SR, Wong TC, Tan CW and Yao K (2013) Piezoelectric polymer multilayer on flexible substrate for energy harvesting. IEEE Transactions on Ultrasonics, Ferroelectrics, and Frequency Control 60: 2013-2020.

Zhu D, Almusallam A, Beeby S, Tudor J and Harris N (2010a) A bimorph multi-layer piezoelectric vibration energy harvester. In: PowerMEMS, 1-3rd December 2010. Leuven, Belgium, p. 4 pages.

Zhu D, Beeby S, Tudor J, White N and Harris N (2011) Improving output power of piezoelectric energy harvesters using multilayer structures. Procedia Engineering 25: 199-202.

Zhu M, Worthington E and Tiwari A (2010b) Design study of piezoelectric energy harvesting devices for generation of higher electrical power using a coupled piezoelectric-circuit finite element method. IEEE Transactions on Ultrasonics, Ferroelectrics, and Frequency Control 57: 427-437. 


\section{Constants in the transfer matrix}

In this appendix the constants required in the transfer matrix for a beam element with axial tension or compression are defined.

$$
\begin{array}{r}
H_{1}=\frac{\left(N^{2} / M^{2}\right) \cosh M+\cos N}{f}, \\
H_{2}=\frac{\left(N^{3} / M^{3}\right) \sinh M+\sin N}{f N}, \\
H_{3}=\frac{\cosh M-\cos N}{f M^{2}}, \\
H_{4}=\frac{\sinh M-(M / N) \sin N}{f M^{3}}, \\
H_{5}=\frac{\sinh M+(N / M) \sin N}{f M}, \\
H_{6}=\frac{\cosh M+\left(N^{2} / M^{2}\right) \cos N}{f}, \\
H_{7}=\frac{N^{2} M \sinh M^{3}+N^{3} \sin N}{f}, \\
H_{8}=\frac{M \sinh M-\left(N^{3} / M^{2}\right) \sin N}{f} .
\end{array}
$$

where $f=1+\left(N^{2} / M^{2}\right)$ is a constant and all other terms have previously been defined.

\section{Potential energy expression constants}

In this appendix, constants in the potential energy expression (Equation (29)), $K_{1}$ through to $K_{6}$ are provided. Note how Heaviside Functions are utilised to isolate various sections of the beam. A schematic of the system provided in Figure 19.

$$
\begin{aligned}
K_{1}(s) & =\left(H\left(s-x_{j-1}\right)-H\left(s-x_{j}\right)\right)(E I)_{j} \quad \text { for } \quad j=1: 5 \\
K_{2}(s) & =\left(H\left(s-x_{1}\right)-H\left(s-x_{2}\right)\right)\left(\mu_{s 1} I_{s 1}+2 \mu_{s 1} \sum_{i=1}^{n / 2}\left(I_{t s 1}\right)_{i}\right) \\
& +\left(H\left(s-x_{2}\right)-H\left(s-x_{3}\right)\right)\left(\mu_{s 1} I_{s 1}+2 \mu_{p 1} \sum_{i=1}^{n / 2}\left(I_{p 1}\right)_{i}+2 \mu_{s 1} \sum_{i=1}^{n / 2}\left(I_{t s 1}\right)_{i}\right) \\
& +\left(H\left(s-x_{3}\right)-H\left(s-x_{4}\right)\right)\left(\mu_{s 1} I_{s 1}+2 \mu_{s 1} \sum_{i=1}^{n / 2}\left(I_{t s 1}\right)_{i}\right) \\
K_{3}(s) & =\left(H\left(s-x_{1}\right)-H\left(s-x_{2}\right)\right)\left(\mu_{s 2} I_{s 2}+2 \mu_{s 2} \sum_{i=1}^{n / 2}\left(I_{t s 2}\right)_{i}\right) \\
& +\left(H\left(s-x_{2}\right)-H\left(s-x_{3}\right)\right)\left(\mu_{s 2} I_{s 2}+2 \mu_{p 2} \sum_{i=1}^{n / 2}\left(I_{p 2}\right)_{i}+2 \mu_{s 2} \sum_{i=1}^{n / 2}\left(I_{t s 2}\right)_{i}\right) \\
& +\left(H\left(s-x_{3}\right)-H\left(s-x_{4}\right)\right)\left(\mu_{s 2} I_{s 2}+2 \mu_{s 2} \sum_{i=1}^{n / 2}\left(I_{t s 2}\right)_{i}\right),
\end{aligned}
$$




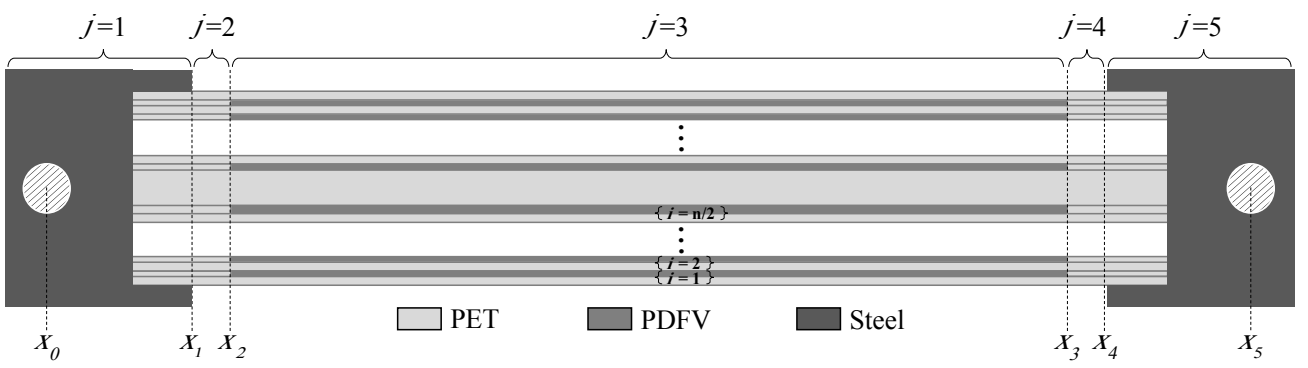

Figure 19. Schematic to show section numbering and notation.

$$
\begin{aligned}
\left(K_{4}(s)\right)_{i} & =\left(H\left(s-x_{2}\right)-H\left(s-x_{3}\right)\right) \\
\times & \left(E_{p} d_{31} b_{p}\right)\left((i-1)\left(t_{t s}+t_{p}\right)+t_{p} / 2+t_{s} / 2\right) \quad \text { for } i=1: n / 2, \\
K_{5}(s) & =\left(H\left(s-x_{1}\right)-H\left(s-x_{1}-x_{2}\right)\right)\left(\frac{\mu_{p 3}}{n t_{p}} \sum_{i=1}^{n / 2}\left(I_{p 3}\right)_{i}\right), \\
K_{6}(s) & =\left(H\left(s-x_{1}\right)-H\left(s-x_{1}-x_{2}\right)\right)\left(\frac{b_{p} \varepsilon_{33}}{t_{p}}\right),
\end{aligned}
$$

where notation has previously been defined in the main body of the paper but partly repeated here for convenience. Subscripts ' $p$ ', ' $s$ ' and ' $t s$ ' refer to piezoelectric, substrate and thin substrate layers respectively, and $n$ is the number of thin PET-PVDF stacks. $\mu_{s 1}, \mu_{s 2}, \mu_{p 1}, \mu_{p 2}$ and $\mu_{p 3}$ are coefficients of material non-linearity. Note how material nonlinearity in the steel connection elements is assumed zero and so no contribution is found in the calculation of $K_{2}$ and $K_{3}$.

$I_{s 1}, I_{s 2}, I_{t s 1}, I_{t s 2}, I_{p 1}, I_{p 2}$ and $I_{p 3}$ are geometry dependant, and for multi-layered compositions are defined by:

$$
\begin{aligned}
I_{s 1} & =b_{s}\left[\frac{\left(\frac{n}{2}\left(t_{t s}+t_{p}\right)+t_{s}-z\right)^{5}}{5}-\frac{\left(\frac{n}{2}\left(t_{t s}+t_{p}\right)-z\right)^{5}}{5}\right], \\
I_{s 2} & =b_{s}\left[\frac{\left(\frac{n}{2}\left(t_{t s}+t_{p}\right)+t_{s}-z\right)^{7}}{7}-\frac{\left(\frac{n}{2}\left(t_{t s}+t_{p}\right)-z\right)^{7}}{7}\right], \\
\left(I_{t s 1}\right)_{i} & =b_{s}\left[\frac{\left((i-1)\left(t_{t s}+t_{p}\right)+t_{t s}-z\right)^{5}}{5}-\frac{\left((i-1)\left(t_{t s}+t_{p}\right)-z\right)^{5}}{5}\right], \\
\left(I_{t s 2}\right)_{i} & =b_{s}\left[\frac{\left((i-1)\left(t_{t s}+t_{p}\right)+t_{t s}-z\right)^{7}}{7}-\frac{\left((i-1)\left(t_{t s}+t_{p}\right)-z\right)^{7}}{7}\right], \\
\left(I_{p 1}\right)_{i} & =b_{p}\left[\frac{\left(t_{t s}+(i-1)\left(t_{t s}+t_{p}\right)+t_{p}-z\right)^{5}}{5}-\frac{\left(t_{t s}+(i-1)\left(t_{t s}+t_{p}\right)-z\right)^{5}}{5}\right], \\
\left(I_{p 2}\right)_{i} & =b_{p}\left[\frac{\left(t_{t s}+(i-1)\left(t_{t s}+t_{p}\right)+t_{p}-z\right)^{7}}{7}-\frac{\left(t_{t s}+(i-1)\left(t_{t s}+t_{p}\right)-z\right)^{7}}{7}\right], \\
\left(I_{p 3}\right)_{i} & =b_{p}\left[\frac{\left(t_{t s}+(i-1)\left(t_{t s}+t_{p}\right)+t_{p}-z\right)^{3}}{3}-\frac{\left(t_{t s}+(i-1)\left(t_{t s}+t_{p}\right)-z\right)^{3}}{3}\right],
\end{aligned}
$$

where $i=1: n / 2$ and $z$ is the location of the neutral axis from the bottom of the device. 


\section{Constants in governing equations of motion}

In this appendix the time-independent constants, $C^{n 1}$ through to $C^{n 15}$, are defined. These constants can be found in the equations of motion, Equations (30) and (31), as multipliers to powers and derivatives of the time-dependant variable, $\eta_{r}$, and the voltage, $V$.

$$
\begin{aligned}
& C_{r}^{n 1}=\left[\int_{0}^{L} W_{q}(s)\left[2 K_{2}(s) W_{r}^{\prime \prime 3}(s)\right]^{\prime \prime} \mathrm{d} s\right] \\
& C_{r}^{n 2}=\left[\int_{0}^{L} W_{q}(s)\left[\left(K_{1}(s) W_{r}^{\prime \prime}(s) W_{r}^{\prime}(s)\right)^{\prime} W_{r}^{\prime}(s)\right]^{\prime} \mathrm{d} s\right] \\
& C_{r}^{n 3}=\left[\int_{0}^{L} W_{q}(s)\left[3 K_{3}(s) W_{r}^{\prime \prime 5}(s)\right]^{\prime \prime} \mathrm{d} s\right] \\
& C_{r}^{n 4}=\left[\int_{0}^{L} W_{q}(s)\left[W_{r}^{\prime}(s) \int_{L}^{s} m(s) \int_{0}^{s}\left(W_{r}^{\prime \prime}(s) W_{r}^{\prime}(s)\right) \mathrm{d} s \mathrm{~d} s\right]^{\prime} \mathrm{d} s\right] \\
& C_{r}^{n 5}=\left[\int_{0}^{L} W_{q}(s)\left[W_{r}^{\prime}(s) \int_{L}^{s} m(s) \int_{0}^{s}\left(W_{r}^{\prime 2}(s)\right) \mathrm{d} s \mathrm{~d} s\right]^{\prime} \mathrm{d} s\right] \\
& \left(C_{r}^{n 6}\right)_{i}=\left[\int_{0}^{L} W_{q}(s)\left[\left(K_{4}(s)\right)_{i}\right]^{\prime \prime} \mathrm{d} s\right] \\
& C_{r}^{n 7}=\left[\int_{0}^{L} W_{q}(s)\left[\left(K_{5}(s)\right) W_{r}^{\prime \prime}(s)\right]^{\prime \prime} \mathrm{d} s\right] \\
& \left(C_{r}^{n 8}\right)_{i}=\left[\int_{0}^{L} W_{q}(s)\left[\left(K_{4}(s)\right)_{i} W_{r}^{\prime \prime}(s) W_{r}^{\prime}(s)\right]^{\prime} \mathrm{d} s\right] \\
& -\left[\int_{0}^{L} W_{q}(s)\left[\frac{1}{2}\left(K_{4}(s)\right)_{i} W_{r}^{\prime 2}(s)\right]^{\prime \prime} \mathrm{d} s\right] \\
& C_{r}^{n 9}=\left[\int_{0}^{L} W_{q}(s)\left[\left(\left(K_{5}(s)\right) W_{r}^{\prime \prime}(s) W_{r}^{\prime}(s)\right)^{\prime} W_{r}^{\prime}(s)\right]^{\prime} \mathrm{d} s\right] \\
& C_{r}^{n 10}=\left[\iiint_{V_{s}} \rho_{s} W_{q}(s) \mathrm{d} V+\iiint_{V_{p}} \rho_{p} W_{q}(s) \mathrm{d} V\right] \\
& C_{r}^{n 11}=\left[\int_{0}^{L} K_{5}(s) W_{r}^{\prime \prime 2}(s) \mathrm{d} s\right] \\
& C_{r}^{n 12}=\left[\int_{0}^{L} 2 K_{5}(s)\left(W_{r}^{\prime \prime 2}(s) W_{r}^{\prime 2}(s)\right) \mathrm{d} s\right] \\
& \left(C_{r}^{n 13}\right)_{i}=\left[\int_{0}^{L}\left(K_{4}(s)\right)_{i} W_{r}^{\prime \prime}(s) \mathrm{d} s\right] \\
& \left(C_{r}^{n 14}\right)_{i}=\left[\int_{0}^{L} 3\left(K_{4}(s)\right)_{i}\left(\frac{1}{2} W_{r}^{\prime \prime}(s) W_{r}^{\prime 2}(s)\right) \mathrm{d} s\right] \\
& C_{r}^{n 15}=\left[\int_{0}^{L} K_{6}(s) \mathrm{d} s\right]
\end{aligned}
$$


In the above expressions, $W_{q}$ and $W_{r}$ are the mass normalised mode shapes of the $q^{\text {th }}$ and $r^{\text {th }}$ mode respectively. 